\title{
Approximate Input Reconstruction for Diagnosing Aircraft Control Surfaces
}

\author{
Haoyun Fu, ${ }^{*}$ Siddharth Kirtikar, ${ }^{\dagger}$ Elena Zattoni, ${ }^{\ddagger}$ Harish Palanthandalam-Madapusi, ${ }^{\S}$ \\ and Dennis S. Bernstein
}

\begin{abstract}
An approximate input reconstruction algorithm is used to reconstruct unknown inputs, which are then used for fault detection. The approximate input reconstruction algorithm is a least squares algorithm that estimates both the unknown initial state and input history. The estimated inputs are then compared to the commanded values and sensor values to assess the health of actuators and sensors. This approach is applied to the longitudinal and lateral dynamics of NASA's Generic Transport Model. The input reconstruction algorithm can be used for systems with minimum-phase or nonminimum-phase zeros; however, minimum-phase zeros entail an additional delay in reconstructing inputs, while zeros on the unit circle yield persistent estimation errors and thus poor input reconstruction regardless of the delay.
\end{abstract}

\section{Introduction}

Fault diagnosis or fault detection $\left[^{1-5}\right]$ refers to the ability to determine the presence of hardware failures. One approach to detecting hardware failures is to use input observers to reconstruct inputs to the system based on a dynamic model and measurements. The estimated inputs are then compared to the commanded inputs to assess the health of sensors and actuators. This approach is based on system inversion techniques developed for either input observers (left inversion) or preview control (right inversion) $\left[{ }^{6-17}\right]$.

One difficulty that arises in system inversion is the presence of zeros. If the system has no zeros, then input reconstruction is possible even if the initial state is unknown and nonzero [ $\left.{ }^{17}\right]$. However, if the system has zeros, then there exists an initial state such that, for some nonzero input, the output is identically zero. Therefore, exact input reconstruction is impossible if the system has at least one zero. For a minimum-phase zero, the unobservable input decays, and thus input observation is asymptotically possible, which means that more recent inputs can be reconstructed more accurately than earlier inputs. Alternatively, minimum-phase zeros can be cancelled through filtering. On the other hand, for a nonminimum-phase zero, the unobservable input increases without bound, and thus input observation is possible asymptotically backward in time, that is, noncausally for large data sets. The increased delay in input reconstruction is thus an unavoidable impediment due to nonminimum-phase zeros. Finally, if the zero lies on the unit disk, then the unobservable input is persistent (non-decaying in both forward and backward time) and thus input reconstruction is not feasible.

In recent work $\left[{ }^{18}\right]$, we applied the input reconstruction approach developed in $\left[{ }^{17}\right]$ to aircraft longitudinal dynamics. The results of $\left[{ }^{17,18}\right]$ assume that input reconstruction occurs after a single measurement delay, that is, 1-step input reconstruction. The contribution of the present paper is to develop input reconstruction techniques for $l$-step input reconstruction, where the increase in the delay needed for input reconstruction takes into account the relative degree of the system. This issue is nontrivial since, for multi-input, multioutput systems, the relative degree may be different for different input channels. As in $\left[{ }^{18}\right]$, we estimate the input by using a least squares solution of the input-output relation. The resulting estimate is unable to

\footnotetext{
* Department of Aerospace Engineering, The University of Michigan, Ann Arbor, MI 48109-2140

$\dagger$ Graduate Research Assistant, Department of Aerospace Engineering The University of Michigan, Ann Arbor, MI 481092140, kirtikar@umich.edu

${ }^{\ddagger}$ Professor, University of Bologna, Bologna, Italy

$\S$ Assistant Professor, Department of Mechanical and Aerospace Engineering, Syracuse University, Syracuse, NY

I Professor, Department of Aerospace Engineering The University of Michigan, Ann Arbor, MI 48109-2140, dsbaero@umich.edu
} 
estimate input components in the null space of the coefficient matrix, which corresponds to the unobservable input component due to the presence of invariant zeros. We apply this algorithm to the linearized longitudinal and lateral dynamics of NASA's GTM $\left[{ }^{20-22}\right]$ in order to estimate control-surface deflections. These estimates can be used to detect faults in the actuator or linkage sensor associated with the control surface.

The present paper goes beyond $\left.{ }^{18}\right]$ in the way that the least squares method is applied. First, unlike $\left[{ }^{18}\right]$, we do not assume that an estimate of the observability matrix is available, but rather we use the eigensystem realization algorithm (ERA) $\left.{ }^{19}\right]$ to construct this matrix based on Markov parameters. This approach is more realistic in practice when only Markov parameters are available and has the additional benefit of reducing the condition number of the observability matrix in the least squares procedure.

\section{Faults in an Aircraft Dynamic System}

We consider the aircraft elevator and engine subsystems, which can potentially undergo various faults. The elevator is assumed to have a local sensor, called the linkage sensor, which provides a measurement of the deflection of the linkage that drives the elevator. Consequently, the elevator subsystem can undergo various faults; for example, the control surface may be damaged, the linkage may be damaged, or the linkage sensor may malfunction.

With notation defined in Table 1, Figure 1 shows the various signals that are used for fault diagnosis. Specifically, $\delta e_{k, \text { com }}$ is the command to the elevator, $\delta \hat{e}_{k}$ is the reconstructed (estimated) value of the elevator deflection, and $l_{k, \mathrm{msmt}}$ is the signal from the linkage sensor.

\begin{tabular}{|c|c|}
\hline Notation & Description \\
\hline$\delta e_{k, \text { com }}$ & commanded elevator input \\
\hline$\delta e_{k, \text { act }}$ & actual elevator output \\
\hline$l_{k, \text { act }}$ & actual linkage output \\
\hline$l_{k, \mathrm{msmt}}$ & linkage sensor measurement \\
\hline$\delta \hat{e}_{k}$ & estimated elevator input \\
\hline$\delta T_{k, \mathrm{com}}$ & commanded engine input \\
\hline$\delta T_{k, \text { act }}$ & actual engine output \\
\hline$\delta T_{k, \mathrm{msmt}}$ & actual RPM sensor output \\
\hline$\delta \hat{T}_{k}$ & estimated engine input \\
\hline
\end{tabular}

Table 1: Notation for signals used to identify faults

Discrepancies between these signals suggest the possibility of various types of failures. Figure 2 shows the analogous signals for engine fault diagnosis.

We consider the possibility that the linkage can fail in various ways. In particular, we consider linkage faults that include saturation, rate saturation, deadzone, and stuck. In addition, linkage sensor faults include bias, drift, stuck, and calibration error. These faults are listed in Table 2, where $b$ is a constant and $c$ is a positive number.

\begin{tabular}{|c|c|}
\hline Linkage Sensor Fault & Description \\
\hline Bias & $l_{k, \mathrm{msmt}}=l_{k, \text { act }}+b$ \\
\hline Drift & $l_{k, \mathrm{msmt}}=l_{k, \mathrm{act}}+k c$ \\
\hline Stuck & $l_{k, \mathrm{msmt}}=b$ \\
\hline Scale Factor Error & $l_{k, \mathrm{msmt}}=l_{k, \mathrm{act}} b$ \\
\hline
\end{tabular}

Table 2: Linkage sensor faults, where $b$ and $c$ are constants.

The elevator control surface can fail in various ways. For example, the elevator may become stuck in a single, fixed deflection. Alternatively, the elevator may become deformed or damaged, resulting in a loss of control effectiveness. Detection of these faults is relevant to health monitoring to mitigate loss of control system performance.

For the elevator, we detect faults during flight by comparing the commanded input $\delta e_{k, \text { com, the measured }}$ 


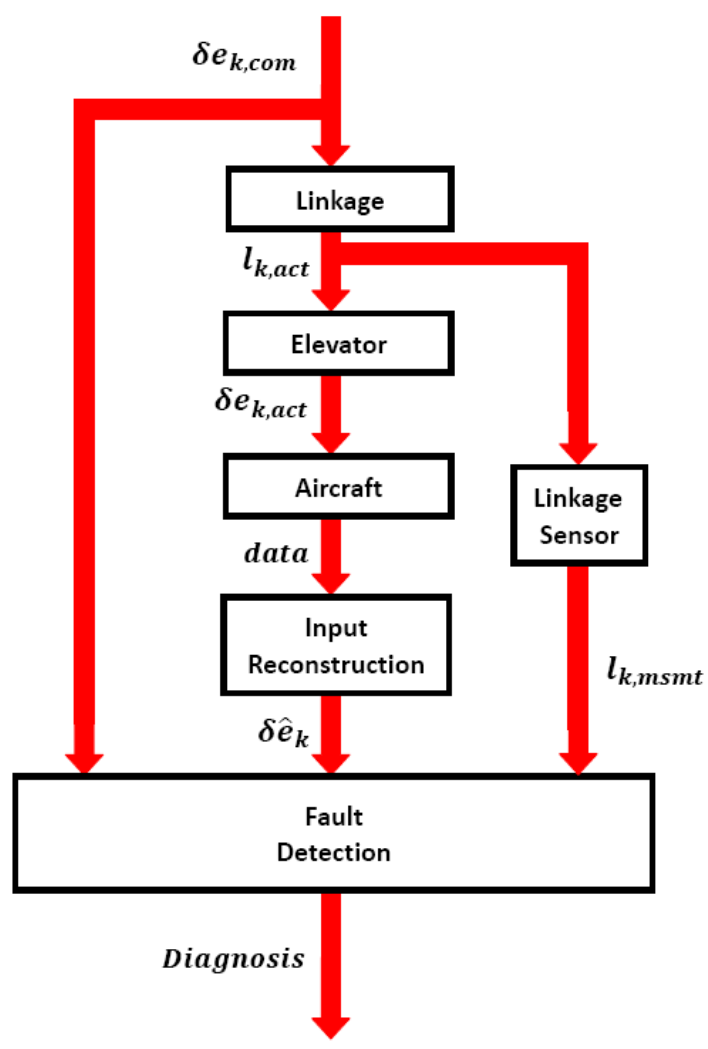

Figure 1: Signals used for elevator fault detection

input $l_{k, \mathrm{msmt}}$ from the linkage sensor, and the estimated input $\delta \hat{e}_{k}$ from input reconstruction. Table 3 shows the logic by which these signals are compared. For example, if the linkage measurement differs from both the commanded elevator deflection and the estimated elevator deflection, then we can conclude that the linkage sensor is faulty but that the linkage and elevator are both operational. In practice, all of these signals are noisy, and thus error criteria are needed. For conceptual simplicity, Table 3 is stated in terms of equality and inequality of signals. A similar fault analysis can be applied to other control surfaces and the engine.

\section{III. l-Delay Input Reconstruction with Known Initial State}

Consider the linear discrete-time system

$$
\begin{aligned}
x_{k+1} & =A x_{k}+B u_{k}, \\
y_{k} & =C x_{k}+D u_{k},
\end{aligned}
$$

where $x_{k} \in \mathbb{R}^{n}, u_{k} \in \mathbb{R}^{m}, y_{k} \in \mathbb{R}^{p}, A \in \mathbb{R}^{n \times n}, B \in \mathbb{R}^{n \times m}, C \in \mathbb{R}^{p \times n}$ and $D \in \mathbb{R}^{p \times m}$. We assume that $(A, B, C)$ is minimal. The $p \times m$ transfer function of $(1),(2)$ is $G(z)=C\left(z I_{n}-A\right)^{-1} B+D$. For each nonnegative integer $r$, we define the output sequence $Y_{[k: k+r]} \in \mathbb{R}^{p(r+1)}$ and the input sequence $U_{[k: k+r]} \in \mathbb{R}^{m(r+1)}$ by

$$
Y_{[k: k+r]} \triangleq\left[\begin{array}{c}
y_{k} \\
y_{k+1} \\
\vdots \\
y_{k+r}
\end{array}\right], U_{[k: k+r]} \triangleq\left[\begin{array}{c}
u_{k} \\
u_{k+1} \\
\vdots \\
u_{k+r}
\end{array}\right]
$$




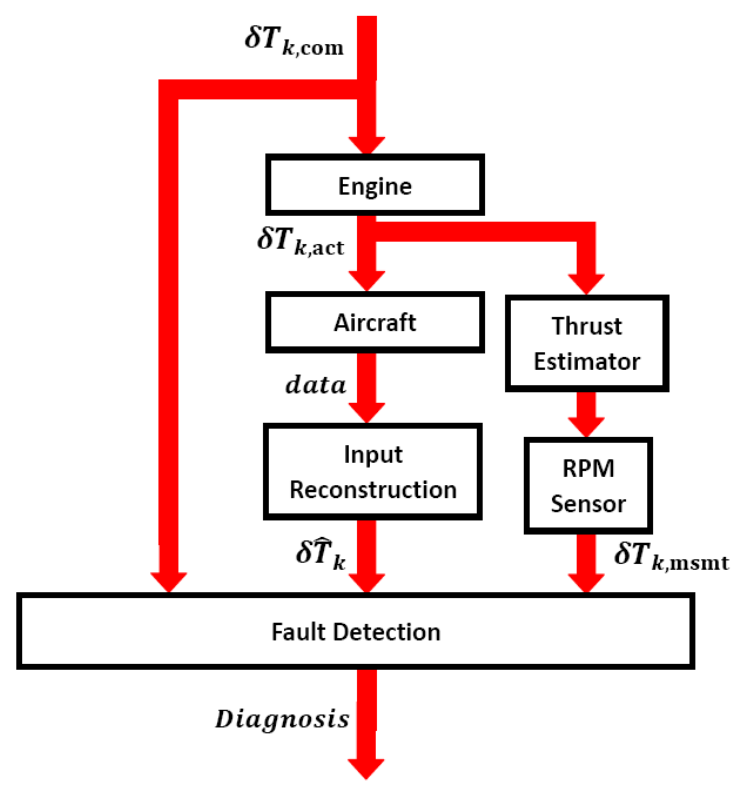

Figure 2: Signals used for engine fault detection

\begin{tabular}{|c|c|c|c|c|}
\hline Case & Condition & $\begin{array}{c}\text { Linkage } \\
\text { Sensor }\end{array}$ & Linkage & Elevator \\
\hline \hline 1 & $\delta e_{k, \mathrm{com}}=\delta \hat{e}_{k}=l_{k, \mathrm{msmt}}$ & $\checkmark$ & $\checkmark$ & $\checkmark$ \\
\hline 2 & $\delta e_{k, \mathrm{com}}=\delta \hat{e}_{k} \neq l_{k, \mathrm{msmt}}$ & Faulty & $\checkmark$ & $\checkmark$ \\
\hline 3 & $\delta e_{k, \mathrm{com}} \neq \delta \hat{e}_{k}=l_{k, \mathrm{msmt}}$ & $\checkmark$ & Faulty & $\checkmark$ \\
\hline 4 & $\delta e_{k, \mathrm{com}}=l_{k, \mathrm{msmt}} \neq \delta \hat{e}_{k}$ & $\checkmark$ & $\checkmark$ & Faulty \\
\hline 5 & $\begin{array}{c}\delta e_{k, \mathrm{com}} \neq \delta \hat{e}_{k} \text { and } \\
\delta e_{k, \mathrm{com}} \neq l_{k, \mathrm{msmt}}\end{array}$ & \multicolumn{3}{|c}{ Combination of Cases $2,3,4$} \\
\hline
\end{tabular}

Table 3: Fault detection analysis for the elevator

The objective is to use measurements of the output vector $Y_{\left[0: r_{1}\right]}$ to determine the initial state $x_{0}$ and the input vector $U_{\left[0: r_{2}\right]}$, where $r_{1}>r_{2} \geq 0$. Note that $Y_{[0: r]}, U_{[0: r]}$, and $x_{0}$ are related by

$$
Y_{[0: r]}=\Gamma_{r} x_{0}+M_{r, r} U_{[0: r]}=\Psi_{r, r}\left[\begin{array}{c}
x_{0} \\
U_{[0: r]}
\end{array}\right],
$$

where, for $r_{1}>r_{2} \geq 0$, that matrices $\Gamma_{r_{1}} \in \mathbb{R}^{p\left(r_{1}+1\right) \times n}, M_{r_{1}, r_{2}} \in \mathbb{R}^{p\left(r_{1}+1\right) \times m\left(r_{2}+1\right)}$, and $\Psi_{r_{1}, r_{2}} \in \mathbb{R}^{p\left(r_{1}+1\right) \times\left[n+m\left(r_{2}+1\right)\right]}$ are defined by

$$
\Gamma_{r_{1}} \triangleq\left[\begin{array}{c}
C \\
C A \\
C A^{2} \\
\vdots \\
C A^{r_{1}}
\end{array}\right], \quad M_{r_{1}, r_{2}} \triangleq\left[\begin{array}{cccc}
H_{0} & 0 & \ldots & 0 \\
H_{1} & H_{0} & \ddots & \vdots \\
H_{2} & H_{1} & \ddots & 0 \\
\vdots & \vdots & \ddots & H_{0} \\
\vdots & \vdots & \vdots & \vdots \\
H_{r_{1}} & H_{r_{1}-1} & \ldots & H_{r_{1}-r_{2}}
\end{array}\right]
$$


and

$$
\Psi_{r_{1}, r_{2}} \triangleq\left[\Gamma_{r_{1}} M_{r_{1}, r_{2}}\right]
$$

and where

$$
H_{k} \triangleq \begin{cases}D, & k=0, \\ C A^{k-1} B, & k \geq 1,\end{cases}
$$

denotes the Markov parameters of the system. Throughout this paper, $d \geq 0$ denotes the relative degree of $G(z)$, that is, the smallest nonnegative integer $i$ such that $H_{i} \neq 0$.

Definition 1. Let $l$ be a nonnegative integer. Then $G(z)$ is l-delay left invertible and the $m \times p$ proper transfer function $G_{l}(z)$ is an l-delay left inverse of $G(z)$ if $G_{l}(z) G(z)=z^{-l} I_{m}$ for almost all $z \in \mathbb{C}$. In this case, the smallest nonnegative integer $l_{0}$ for which $G(z)$ is $l_{0}$-delay left invertible is the minimal delay of $G$. Finally, $G$ is left invertible if there exists a nonnegative integer $q$ such that $G$ is $q$-delay left invertible.

Note that if $G(z)$ is $l$-delay left invertible, then $G(z)$ is $r$-delay left invertible for all $r \geq l$.

Theorem 1. The following statements are equivalent:

(i) $G(z)$ is left invertible.

(ii) The normal rank of $G(z)$ is $m$.

(iii) The normal rank of $\left[\begin{array}{cc}z I-A & -B \\ C & D\end{array}\right]$ is $n+m$.

Theorem 1 shows that if $G(z)$ is $l$-delay left invertible, then $G_{l}(z) G(z)=z^{-l} I_{m}$ holds for all $z \in \mathbb{C}$ except the poles of $G(z)$ and $G_{l}(z)$ and, if $l \geq 1, z=0$.

Now, for $r \geq l \geq 0$, partition $M_{r, r}$ as

$$
M_{r, r}=\left[\begin{array}{ll}
M_{r, r-l} & \tilde{M}_{r, l}
\end{array}\right]
$$

where $\tilde{M}_{r, l} \in \mathbb{R}^{p(r+1) \times m l}$. Noting that

$$
\Psi_{r, r}=\left[\begin{array}{ll}
\Gamma_{r} & M_{r, r}
\end{array}\right]=\left[\begin{array}{lll}
\Gamma & M_{r, r-l} & \tilde{M}_{r, l}
\end{array}\right]=\left[\begin{array}{ll}
\Psi_{r, r-l} & \tilde{M}_{r, l}
\end{array}\right],
$$

it follows that

$$
\begin{aligned}
Y_{[0: r]} & =\Gamma_{r} x_{0}+M_{r, r-l} U_{[0: r-l]}+\tilde{M}_{r, l} U_{[r-l+1: r]} \\
& =\Psi_{r, r-l}\left[\begin{array}{c}
x_{0} \\
U_{[0: r-l]}
\end{array}\right]+\tilde{M}_{r, l} U_{[r-l+1: r]} .
\end{aligned}
$$

where

$$
\tilde{M}_{r, l}= \begin{cases}0_{p(r+1) \times 0}, & l=0 \\
{\left[\begin{array}{c}
0_{p(r-l+1) \times m l} \\
M_{l-1, l-1}
\end{array}\right],} & l \geq 1 .\end{cases}
$$

and thus, for all $l \geq 1$,

$$
\operatorname{rank}\left(\tilde{\mathrm{M}}_{r, l}\right)=\operatorname{rank}\left(\mathrm{M}_{l-1, l-1}\right)
$$

Let $\mathcal{R}$ denote range.

Theorem 2. Let $l$ be a nonnegative integer. Then the following statements are equivalent:

(i) $G(z)$ is $l$-delay left invertible. 
(ii) There exists a matrix $K \in \mathbb{R}^{m \times p(l+1)}$ such that

$$
K M_{l, l}=\left[\begin{array}{llll}
I_{m} & 0 & \cdots & 0
\end{array}\right]
$$

(iii) $u_{0}$ is uniquely determined by $Y_{[0: l]}$ and $x_{0}$.

(iv) $\mathcal{N}\left(M_{l, l}\right) \subseteq \mathcal{R}\left(\left[\begin{array}{c}0_{m \times m l} \\ I_{m l}\end{array}\right]\right)$

(v) $M_{l, 0}$ has full column rank, and

$$
\mathcal{R}\left(M_{l, 0}\right) \cap \mathcal{R}\left(\tilde{M}_{l, l}\right)=\{0\} .
$$

(vi) $m= \begin{cases}\operatorname{rank}\left(M_{0,0}\right), & l=0 \\ \operatorname{rank}\left(M_{l, l}\right)-\operatorname{rank}\left(M_{l-1, l-1}\right), & l \geq 1\end{cases}$

Theorem 2 implies that $G(z)$ is $l$-delay left invertible if and only if each of the first $m$ columns of $M_{l, l}$ is linearly independent of the remaining columns of $M_{l, l}$.

Note that if $G(z)$ is SISO, then $z^{-d} G^{-1}(z)$ is exactly proper, and thus $l_{0}=d$. The following result gives a necessary and sufficient condition for $l_{0}=d$.

Proposition 1. Assume that $G(z)$ is $l$-delay left invertible, and let $l_{0}$ denote the minimal delay of $G(z)$. Then,

$$
\begin{gathered}
\operatorname{rank}\left[\begin{array}{ccc}
H_{0} & \cdots & H_{l}
\end{array}\right] \geq m, \\
\operatorname{rank}\left[\begin{array}{c}
H_{0} \\
H_{1} \\
\vdots \\
H_{l}
\end{array}\right]=m,
\end{gathered}
$$

and $l_{0} \leq n$. Furthermore, $l_{0} \geq d$ with equality if and only if $\operatorname{rank}\left(H_{d}\right)=m$.

Proposition 1 shows that if $G(z)$ is $l$-delay left invertible, then both (14) and (15) are satisfied. However, the converse does not hold.

Theorems 1 and 2 provides necessary and sufficient conditions under which the identity transfer function can be attained by a left inverse after $l$ steps. However, this noncausal left inverse has limited value for input reconstruction for two reasons. First, the transfer function formulation of a dynamical system does not account for the initial condition of the state of a corresponding state space realization. Therefore, we must consider a state space formulation in order to have a more complete picture of the free response of the system, which is present in practice. Furthermore, within a state space formulation, if $G(z)$ has a transmission zero, then $G(z)$ can have a nonzero input such that, for some nonzero initial condition of a corresponding state space model, the output is identically zero. We explore these issues in the following sections.

Theorem 3. Assume that $G(z)$ is $l$-delay left invertible. Then for all $r \geq l, U_{[0: r-l]}$ is uniquely determined by $x_{0}$ and $Y_{[0: r]}$. Furthermore, the unique solution with reconstruction delay $l$ is

$$
U_{[0: r-l]}=\operatorname{row}_{[1:(r-l+1) m]}\left[M_{r, r}^{\dagger}\left(Y_{[0: r]}-\Gamma_{r} x_{0}\right)\right] .
$$

Theorem 3 shows that if $G(z)$ is $l$-delay left invertible then we can achieve input reconstruction with an $l$-step delay from known initial condition $x_{0}$ and output measurements $Y_{[0: r]}$.

\section{IV. l-Delay Input and Initial State Reconstruction with Unknown Initial State}

In this section we take into account the unknown and possibly nonzero initial condition of (1), (2) in order to achieve input and state reconstruction. 
Definition 2. Let $l$ be a nonnegative integer. The system (1), (2) is l-delay input and state observable if there exists $r \geq l$ such that

$$
\mathcal{N}\left(\Psi_{r, r}\right) \subseteq \mathcal{R}\left[\begin{array}{c}
0_{[n+m(r-l+1)] \times l m} \\
I_{l m}
\end{array}\right]
$$

In this case, the smallest nonnegative integer $l_{0}^{\prime}$ for which (1), (2) is $l_{0}^{\prime}$-delay input and state observable is the minimal ISO delay. Furthermore, (1), (2) is input and state observable if there exists a nonnegative integer $q$ such that (1), (2) is $q$-delay input and state observable.

In Definition $2, r+1$ is the length of the data window required to reconstruct the initial state and input. Specifically, condition (17) implies that in (4) if $Y_{[0: r]}=0$, that is, $\left[\begin{array}{c}x_{0} \\ U_{[0: r]}\end{array}\right] \in \mathcal{N}\left(\Psi_{r, r}\right)$, then $\left[\begin{array}{c}x_{0} \\ U_{[0: r-l]}\end{array}\right]=0$, and thus $x_{0}$ and $U_{[0: r]}$ can be uniquely reconstructed from $Y_{[0: r]}$.

The following result provides necessary and sufficient conditions for $l$-delay input and state observability.

Proposition 2. Let $l$ be a nonnegative integer. The system (1), (2) is $l$-delay input and state observable if and only if there exists $r \geq l$ such that both of the following statements hold:

(i) $\operatorname{rank}\left(\Psi_{r, r}\right)=\operatorname{rank}\left(\Psi_{r, r-l}\right)+\operatorname{rank}\left(\tilde{M}_{r, l}\right)$.

(ii) $\Psi_{r, r-l}$ has full column rank.

Proposition 3. Assume that (1), (2) is $l$-delay input and state observable. Then $G(z)$ is $l$-delay left invertible. Furthermore,

$$
l_{0} \leq l_{0}^{\prime}
$$

Proposition 4. The following statements are equivalent:

(i) $G(z)$ is left invertible and, for all $r \geq n, \mathcal{R}\left(\Gamma_{r}\right) \cap \mathcal{R}\left(M_{r, r}\right)=\{0\}$.

(ii) For all $\zeta \in \mathbb{C}$, rank $\left[\begin{array}{cc}\zeta I-A & -B \\ C & D\end{array}\right]=n+m$.

Proposition 5. The following statements are equivalent:

(i) (1), (2) is l-delay input and state observable.

(ii) $G(z)$ is $l$-delay left invertible and (1), (2) has no invariant zeros.

Corollary 1. Assume that $G(z)$ left invertible and has no invariant zeros. Then

$$
l_{0}=l_{0}^{\prime} \leq n .
$$

If, in addition, $m=p=1$, then $l_{0}=l_{0}^{\prime}=n$ and, for all $r \geq n-1, \operatorname{def}\left(\Psi_{r, r}\right)=n$.

The following result provides necessary conditions for $l$-delay input and state observability.

Proposition 6. Let $l$ be a nonnegative integer, and assume that (1), (2) is $l$-delay input and state observable. Then the following statements hold:

(i) (1), (2) is $k$-delay input and state observable for all $k \geq l$.

(ii) $\operatorname{rank}\left(\Psi_{r, r-l}\right)=\operatorname{rank}\left(\Psi_{r, r-l-1}\right)+m$ for all $r \geq n$.

(iii) $m \leq p$.

(iv) If $m=p$, then $n \leq m l$.

(v) $\operatorname{rank}\left(\Gamma_{n-1}\right)=n$. 
Proposition 7. Assume that (1), (2) is $l$-delay input and state observable and (17) holds for some $q \geq l$. Then, for all $r \geq q, x_{0}$ and $U_{[0: r-l]}$ are uniquely determined by $Y_{[0: r]}$. Furthermore, the unique solution of (8) with reconstruction delay $l$ is given by

$$
\left[\begin{array}{c}
x_{0} \\
U_{[0: r-l]}
\end{array}\right]=\left(Q^{\mathrm{T}} \Psi_{r, r-l}\right)^{\dagger} Q^{\mathrm{T}} Y_{[0: r]},
$$

where $\dagger$ represents the Moore-Penrose generalized inverse, $k \triangleq \operatorname{rank}\left(\tilde{M}_{r, l}\right)$, and the columns of $Q \in$ $\mathbb{R}^{p(r+1) \times[p(r+1)-k]}$ are an orthonormal basis of $\mathcal{N}\left(\tilde{M}_{r, l}^{\mathrm{T}}\right)$.

If the linear system (1), (2) has invariant zeros, then it follows from Proposition 6 that (1), (2) is not $l$-delay input and state observable, that is, for $r \geq l, x_{0}$ and $U_{[0: r-l]}$ cannot be uniquely reconstructed from $Y_{[0: r]}$ alone. Approximate noncausal reconstruction must account for the locations of invariant zeros in the complex plane relative to the unit disk, as shown in Figure 3. If all of the invariant zeros are contained in the open unit disk, then approximate causal reconstruction is possible for sufficiently large data sets and large times $k$. On the other hand, if all of the invariant zeros are contained in the complement of the closed unit disk, then approximate noncausal reconstruction is possible for sufficiently large data sets and small times $k$. Consequently, if all of the invariant zeros are contained in either the open unit disk or the complement of the closed unit disk, then approximate noncausal reconstruction is possible for sufficiently large data sets and small times $k$. Finally, if at least one invariant zero lies on the unit circle, then approximate reconstruction is not possible since a persistent reconstruction error will corrupt the reconstructed inputs.

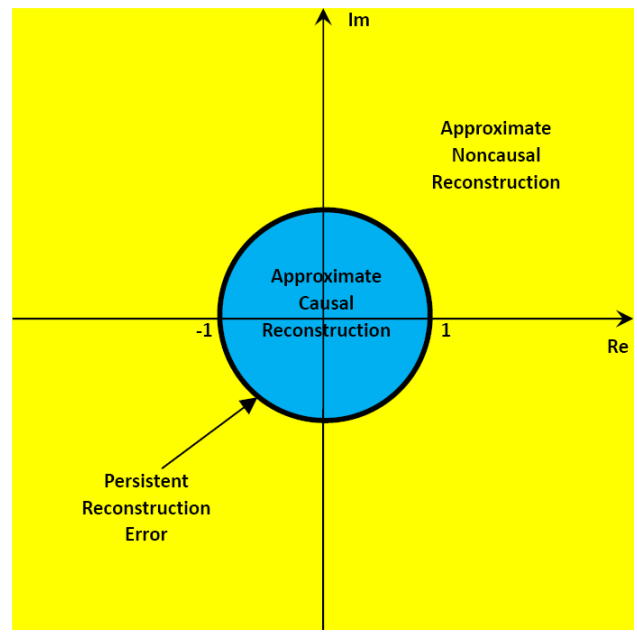

Figure 3: Regions of invariant zeros and their effect on approximate input reconstruction

\section{Approximate Input Reconstruction for SISO Systems with Invariant Zeros}

Definition 3. The l-delay input and state estimate of the system (1), (2) is given by

$$
\left[\begin{array}{c}
\hat{x}_{0} \\
\hat{U}_{[0: r-l]}
\end{array}\right] \triangleq\left(Q^{\mathrm{T}} \Psi_{r, r-l}\right)^{\dagger} Q^{\mathrm{T}} Y_{[0: r]},
$$

where $\dagger$ represents the Moore-Penrose generalized inverse, $k \triangleq \operatorname{rank}\left(\tilde{M}_{r, l}\right)$, and the columns of $Q \in$ $\mathbb{R}^{p(r+1) \times[p(r+1)-k]}$ are an orthonormal basis of $\mathcal{N}\left(\tilde{M}_{r, l}^{\mathrm{T}}\right)$.

In this section, we consider the effect of zeros on the reconstruction algorithm given by the estimate (21). Note that, none of the examples in this section are $l$-delay input and state observable. We consider a realization of the type of (1), (2), where $A, B$ are given by

$$
A=\left[\begin{array}{ccc}
0.6 & -0.22 & 0.096 \\
0.5 & 0 & 0 \\
0 & 0.125 & 0
\end{array}\right], \quad B=\left[\begin{array}{l}
4 \\
0 \\
0
\end{array}\right],
$$


and where various matrices $C$ are used to vary the invariant zeros relative to the unit circle. Note that $x_{0}=\left[\begin{array}{ccc}2 & 0.1 & -1\end{array}\right]$ is used for simulation in examples $1,2,4$ and $x_{0}=\left[\begin{array}{ccc}10 & 5 & 15\end{array}\right]$ is used for simulation in example 3, but knowledge of $x_{0}$ is not assumed to be available for input reconstruction.

Example 1. Consider the minimum-phase transfer function

$$
G(z)=\frac{z-0.5}{(z-0.1)(z-0.2)(z-0.3)},
$$

which has $C=\left[\begin{array}{lll}0 & 0.5 & -2\end{array}\right]$. Figure 4 compares the 2-delay reconstructed input sequence with the actual input sequence. Note that the input reconstruction error is largest for small values of $k$, which corresponds to the fact that the system has a minimum-phase zero.

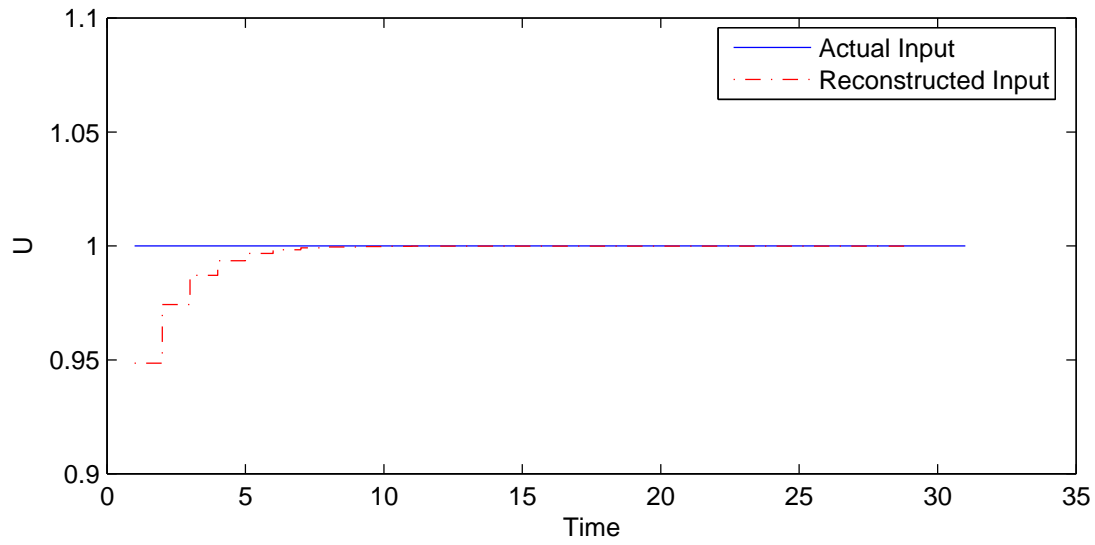

Figure 4: Comparison between the actual input sequence and 2-delay reconstructed input of the system given by Example 1, with the minimum-phase zero 0.5. In this case, the input reconstruction error is monotonically decaying.

Example 2. Consider the transfer function

$$
G(z)=\frac{z-1}{(z-0.1)(z-0.2)(z-0.3)},
$$

which has $C=\left[\begin{array}{lll}0 & 0.5 & -4\end{array}\right]$. Figure 5 compares the 2-delay reconstructed input sequence with the actual input sequence. Note that the input reconstruction error is persistent over the interval, which is a consequence of the fact that the zero is located on the unit circle. In addition, the sign of the error is constant due to the fact that the zero is located at 1.

Example 3. Consider the transfer function

$$
G(z)=\frac{z+1}{(z-0.1)(z-0.2)(z-0.3)},
$$

which has $C=\left[\begin{array}{lll}0 & 0.5 & 4\end{array}\right]$. Figure 6 compares the 2-delay reconstructed input sequence with the actual input sequence. Note that the input reconstruction error is persistent over the interval, which is a consequence of the fact that the zero is located on the unit circle. In addition, the sign of the error alternates at each time step due to the fact that the zero is located at -1 .

Example 4. Consider the nonminimum-phase transfer function

$$
G(z)=\frac{z-3}{(z-0.1)(z-0.2)(z-0.3)},
$$

which has $C=\left[\begin{array}{lll}0 & 0.25 & -6\end{array}\right]$. Figure 7 compares the 2-delay reconstructed input sequence with the actual input sequence. Note that the input reconstruction error is monotonically increasing due to the real nonminimum-phase zero. 


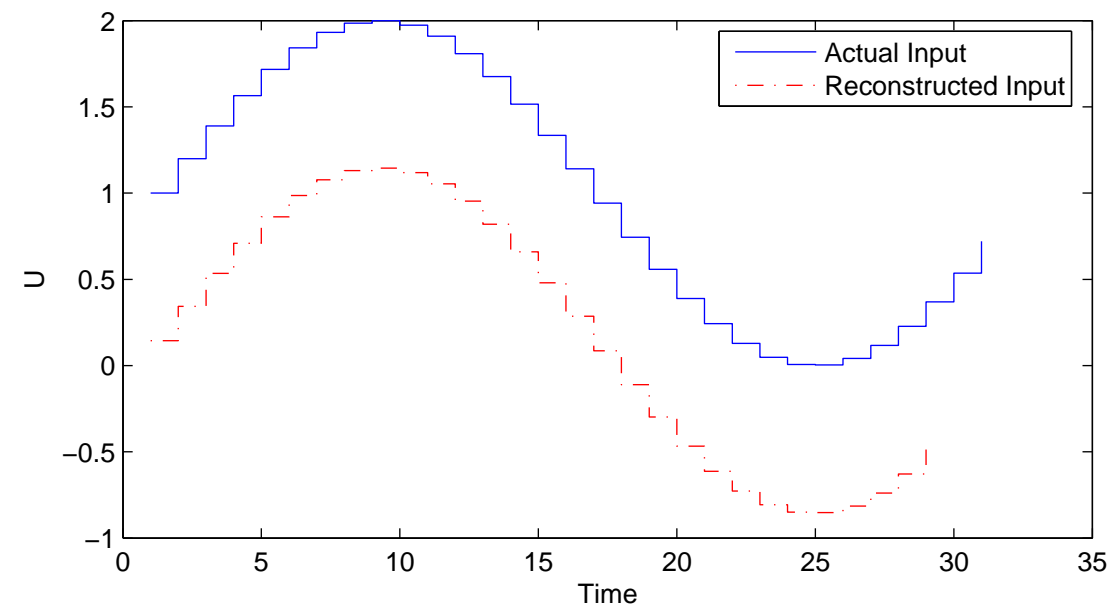

Figure 5: Comparison between the actual input sequence and 2-delay reconstructed input of the system given by Example 2, with zero 1. In this case the input reconstruction error is persistent, in fact, it is constant over the interval.

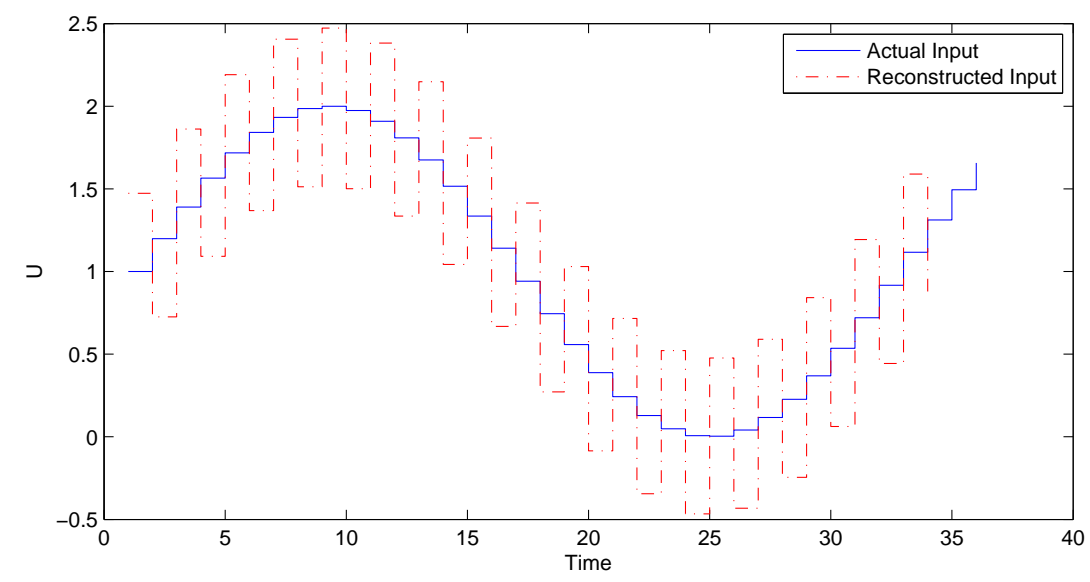

Figure 6: Comparison between the actual input sequence and 2-delay reconstructed input of the system given by Example 3, with zero - 1 . In this case the input reconstruction error is persistent and has alternating sign over the interval. 


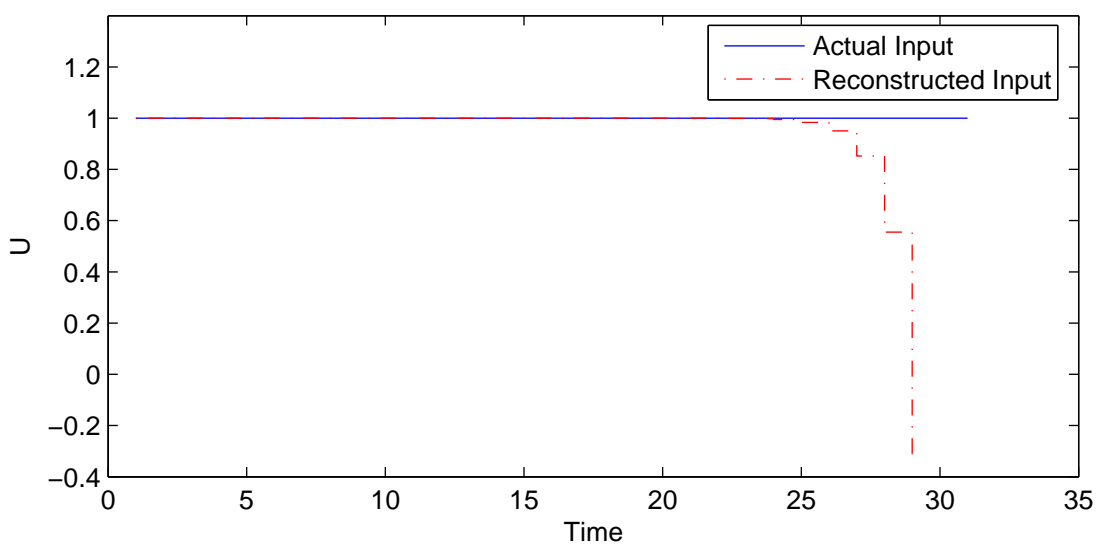

Figure 7: Comparison between the actual input sequence and 2-delay reconstructed input of the system given by Example 4, with nonminimum-phase zero 3. The input reconstruction error is monotonically increasing due to the real nonminimum-phase zero.

\section{Application to GTM}

In this section we use the approximate noncausal input reconstruction algorithm to estimate the input signals for the Generic Transport Model (GTM). In particular, we estimate the aileron, rudder, and elevator deflections using measurements of forward and lateral velocity, as well as roll and yaw rate, which are assumed to be available from flight sensors. The linearized GTM provides the state-space matrices $A, B, C$, and $D$ for flight dynamics once the trimmed flight conditions are specified.

In this case, the state-space form of GTM is

$$
\begin{aligned}
& \dot{X}=A X+B U, \\
& Y=C X+D U,
\end{aligned}
$$

where $X \triangleq\left[\begin{array}{llllllllllll}u & v & w & p & q & r & \text { Lat } & \text { Lon } & \text { Alt } & \phi & \theta & \psi\end{array}\right]^{\mathrm{T}}, U \triangleq\left[\begin{array}{ccc}\delta a & \delta e & \delta r\end{array}\right]^{\mathrm{T}}$, and $Y \triangleq$ $\left[\begin{array}{llll}u & v & p & r\end{array}\right]^{\mathrm{T}}$. The states are $x$-direction velocity $(\mathrm{ft} / \mathrm{s}), y$-direction velocity $(\mathrm{ft} / \mathrm{s}), z$-direction velocity $(\mathrm{ft} / \mathrm{s})$, roll rate $p(\mathrm{rad} / \mathrm{s})$, pitch rate $q(\mathrm{rad} / \mathrm{s})$, yaw rate $r(\mathrm{rad} / \mathrm{s})$, latitude Lat $(\mathrm{ft})$, longitude Lon $(\mathrm{ft})$, altitude $A t l$ (ft), roll angle $\phi(\mathrm{rad})$, pitch angle $\theta(\mathrm{rad})$, and yaw angle psi (rad). The inputs are aileron deflection $\delta a$, elevator deflection $\delta e$ from trim and rudder deflection $\delta r$, while the available measurements are $u, v, p$, and $r$.

Next we compute the Markov parameters $H_{0}=D, H_{i}=C A^{i-1} B, i \geq 1$. We use these matrices to estimate the observability matrix $\Gamma_{r}$ based on the eigensystem realization algorithm (ERA). ${ }^{19}$ The computational steps of ERA are summarized as follows:

1. Construct the $p(r+1) \times m(s+1)$ block-Hankel matrix $\mathcal{H}_{r s}(0)$

$$
\mathcal{H}_{r s}(0) \triangleq\left[\begin{array}{ccccc}
H_{1} & H_{2} & H_{3} & \ldots & H_{s+1} \\
H_{2} & H_{3} & H_{4} & \ldots & H_{s+2} \\
\vdots & \vdots & \vdots & \ddots & \vdots \\
H_{r+1} & H_{r+2} & H_{r+3} & \ldots & H_{r+s+1}
\end{array}\right] .
$$

2. Decompose $\mathcal{H}_{r s}(0)$ using singular value decomposition.

$$
\mathcal{H}_{r s}(0)=P \Sigma Q
$$


where $P \in \mathbb{R}^{p(r+1) \times p(r+1)}$ and $Q \in \mathbb{R}^{m(s+1) \times m(s+1)}$ are both orthogonal matrices, $\Sigma \in \mathbb{R}^{p(r+1) \times m(s+1)}$, and

$$
\Sigma=\left[\begin{array}{cc}
\Sigma_{1} & 0 \\
0 & 0
\end{array}\right]
$$

where $\Sigma_{1} \in \mathbb{R}^{n \times n}$ is a diagonal matrix with positive diagonal entries.

3. Factor $\mathcal{H}_{r s}(0)$ further as

$$
\mathcal{H}_{r s}(0)=P\left[\begin{array}{c}
\Sigma_{1}^{1 / 2} \\
0
\end{array}\right]\left[\begin{array}{ll}
\Sigma_{1}^{1 / 2} & 0
\end{array}\right] Q
$$

Define

$$
L_{r} \triangleq P\left[\begin{array}{c}
\Sigma_{1}^{1 / 2} \\
0
\end{array}\right] \in \mathbb{R}^{p(r+1) \times n},
$$

which is an estimate of $\Gamma_{r}$. The matrix $L_{r}$ is used to form matrix $\Psi_{r, r-l}$ in the following simulations to perform input reconstruction.

The state-space basis of the ERA realization has no physical meaning but is relevant for input reconstruction. We obtain the state-space matrices for the trim condition using an altitude of $625.48 \mathrm{ft}$, an $x$-direction velocity of $95 \mathrm{ft} / \mathrm{s}$, and a pitch angle of $2.52 \mathrm{deg}$. For the system (27)-(28), we apply the approximate noncausal input reconstruction method. The relative degree of the system is $d=1$, and we take $l=2, r=20$. The aileron deflection angle $\delta a$ is chosen to be sinusoidal, and the elevator deflection command $\delta e$ and the rudder deflection command $\delta r$ are chosen to be sawtooth signals. The unknown commands are estimated using (21).

Using Table 3, fault detection is conducted for various fault scenarios. We use the same initial state, and commanded inputs to compare the reconstruction process in each scenarios. Figures 8-17 show the commanded inputs and their 2-delay reconstructed inputs.

Next, we generate erroneous Markov parameter estimates by adding an independent Gaussian random variable to each Markov parameter $H_{i}$ with standard deviation equal to $\frac{H_{i}}{40}$. We then perform the input reconstruction using (21) and these perturbed Markov parameters. Figures 18-25 show the commanded inputs and their 2-delay reconstructed inputs.

\section{Conclusion}

As an extension of approximate causal input reconstruction, we considered an approximate noncausal input reconstruction method where a least squares solution is used in the presence of zeros. For plants with minimum-phase zeros, the input-reconstruction error decays; for nonminimum-phase zeros it grows; and for zeros on the unit circle, it is persistent. We applied this technique to aircraft fault detection, where the objective is to compare the estimated input with the commanded input and the linkage sensor. This comparison provides a technique for online fault detection. We demonstrated the technique for both lateral and longitudinal dynamics. Future work will focus on the effect of model errors and sensor noise on the fault-detection accuracy.

\section{References}

${ }^{1}$ R. K. Douglas and J. I. Speyer. Robust fault detection filter design. Proc. Amer. Contr. Conf., 91-96, 1995.

${ }^{2}$ R. Isermann, Fault Detection and Diagnosis in Engineering Systems. CRC, 1998.

${ }^{3}$ D. Dasgupta, K. KrishnaKumar, D. Wong, and M. Berry, "Negative Selection Algorithm for Aircraft Fault Detection," in Proceedings of Third International Conference on Artificial Immune Systems, pp. 1-13. Springer, 2004.

${ }^{4}$ R. Isermann, Fault Diagnosis Systems: An Introduction form Fault Detection to Fault Tolerance. Springer, 2005.

${ }^{5}$ M. Blanke, M. Kinnaert, J. Lunze, and M. Saroswiecki, Diagnosis and Fault-Tolerant Control. Springer, 2006.

${ }^{6}$ J.L. Massey and M.K. Sain. Inverses of linear sequential circuits. IEEE Transactions of Automatic Control, C-17:330-337, 1968. 

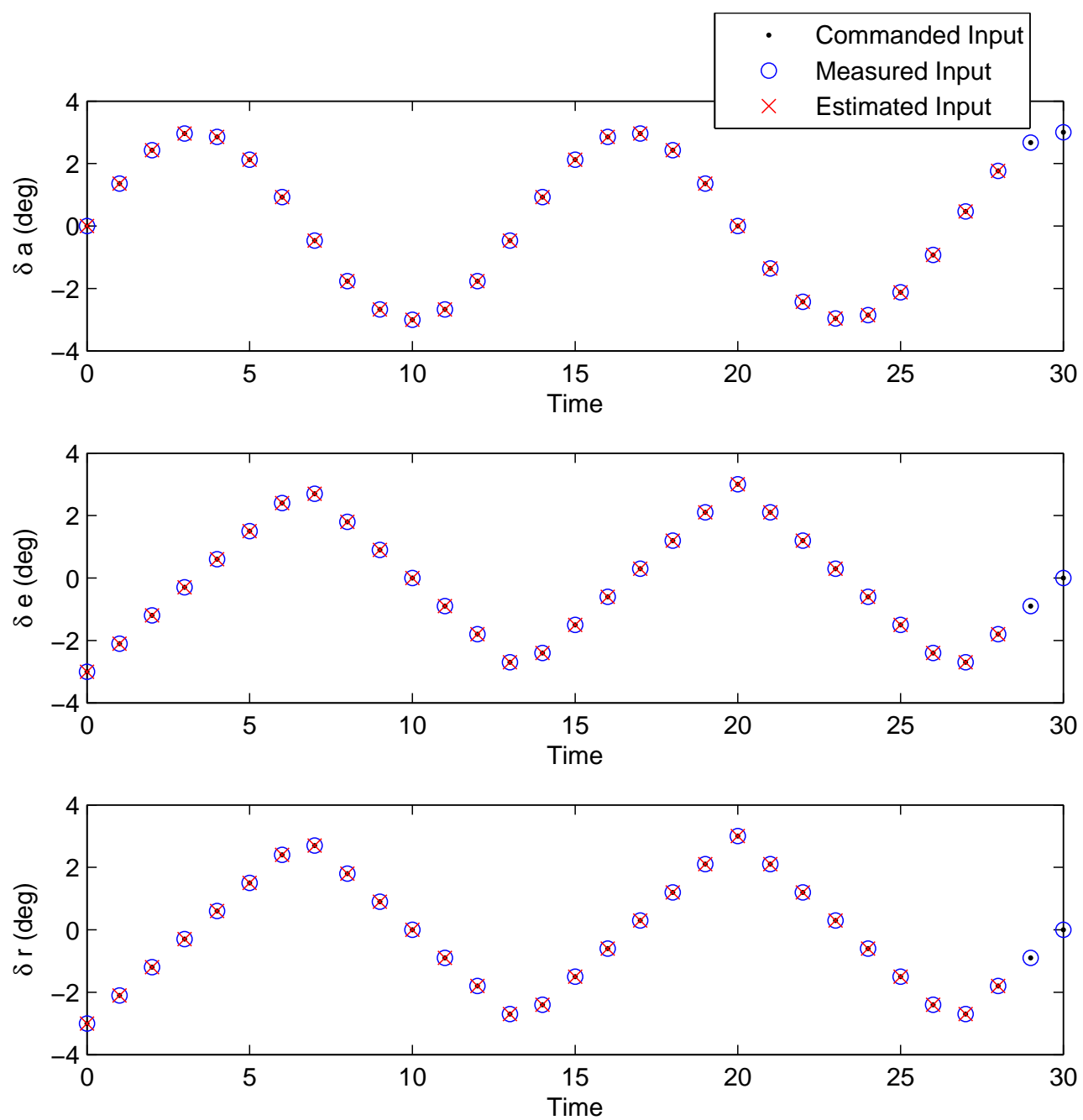

Figure 8: 2-step delay input reconstruction for GTM. Estimates of the unknown inputs $\delta a, \delta e, \delta r$ are obtained by using measurements of the outputs $u, v, p, r$ in the linearized flight model. 

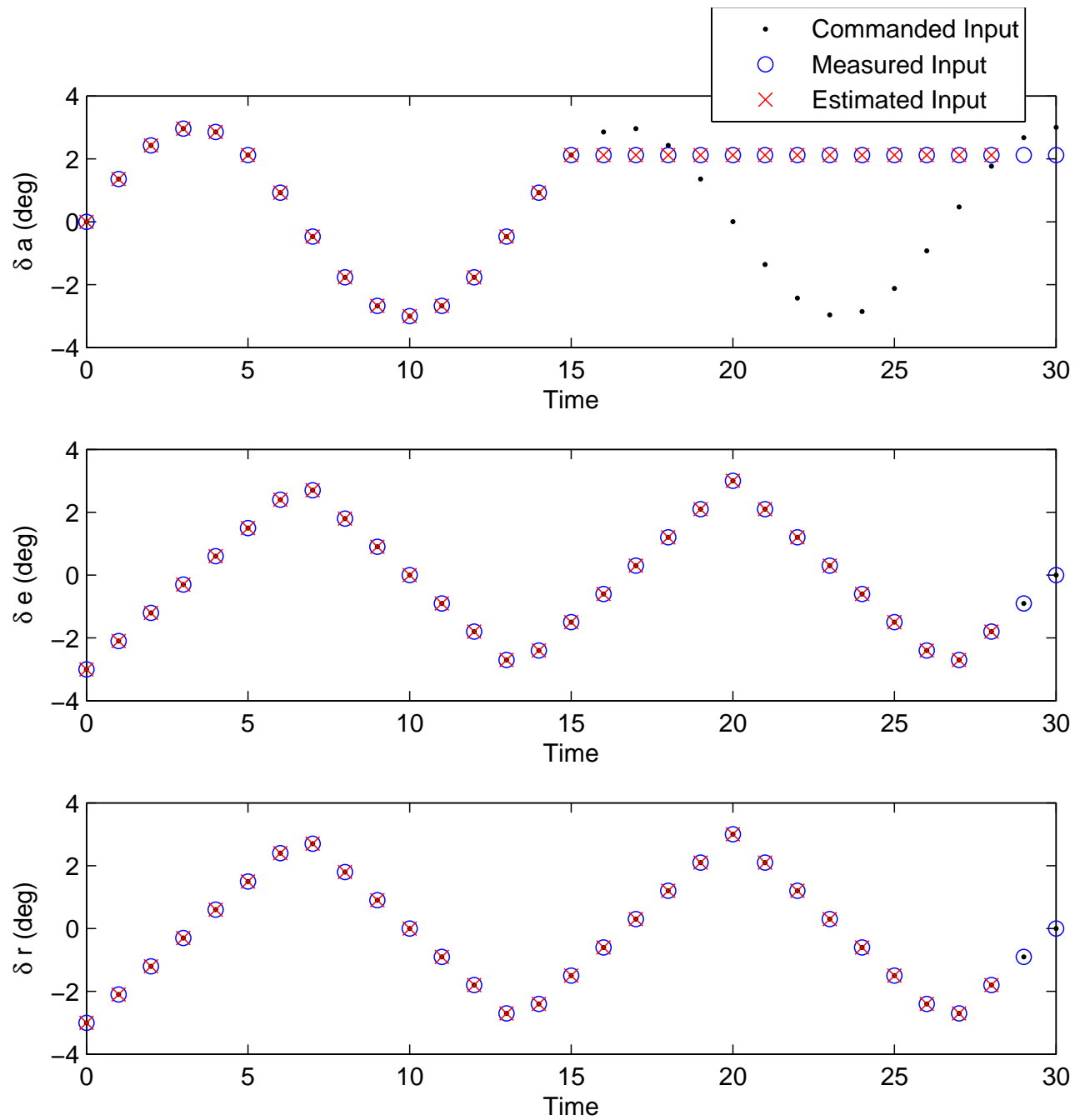

Figure 9: 2-step delay input reconstruction for GTM. Estimates of the unknown inputs $\delta a, \delta e, \delta r$ are obtained by using measurements of the outputs $u, v, p, r$ in the linearized flight model. In this example, the ailerons become stuck at $k=16$. 

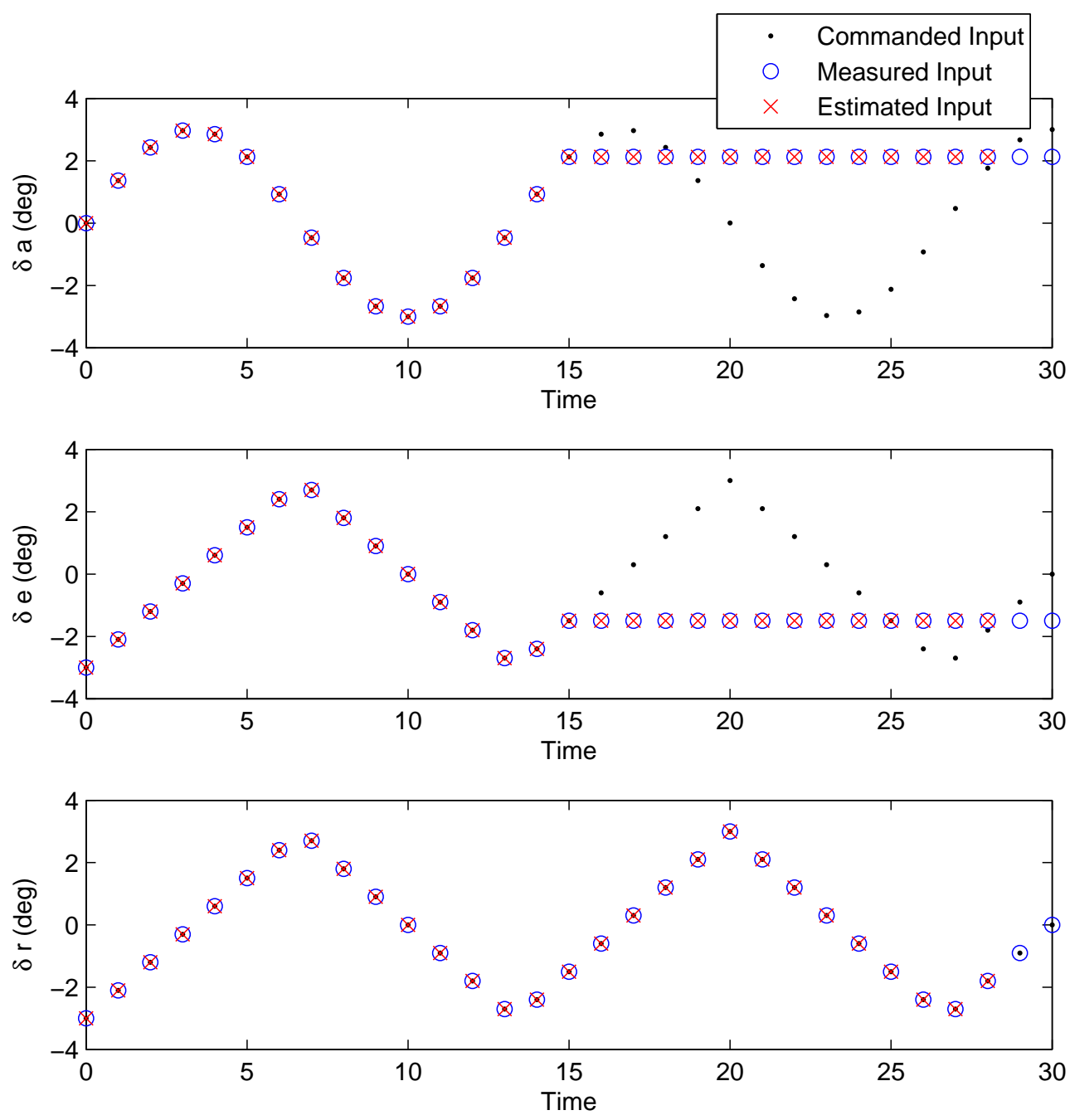

Figure 10: 2-step delay input reconstruction for GTM. Estimates of the unknown inputs $\delta a, \delta e, \delta r$ are obtained by using measurements of the outputs $u, v, p, r$ in the linearized flight model. In this example, the ailerons and elevator both become stuck at $k=16$. 

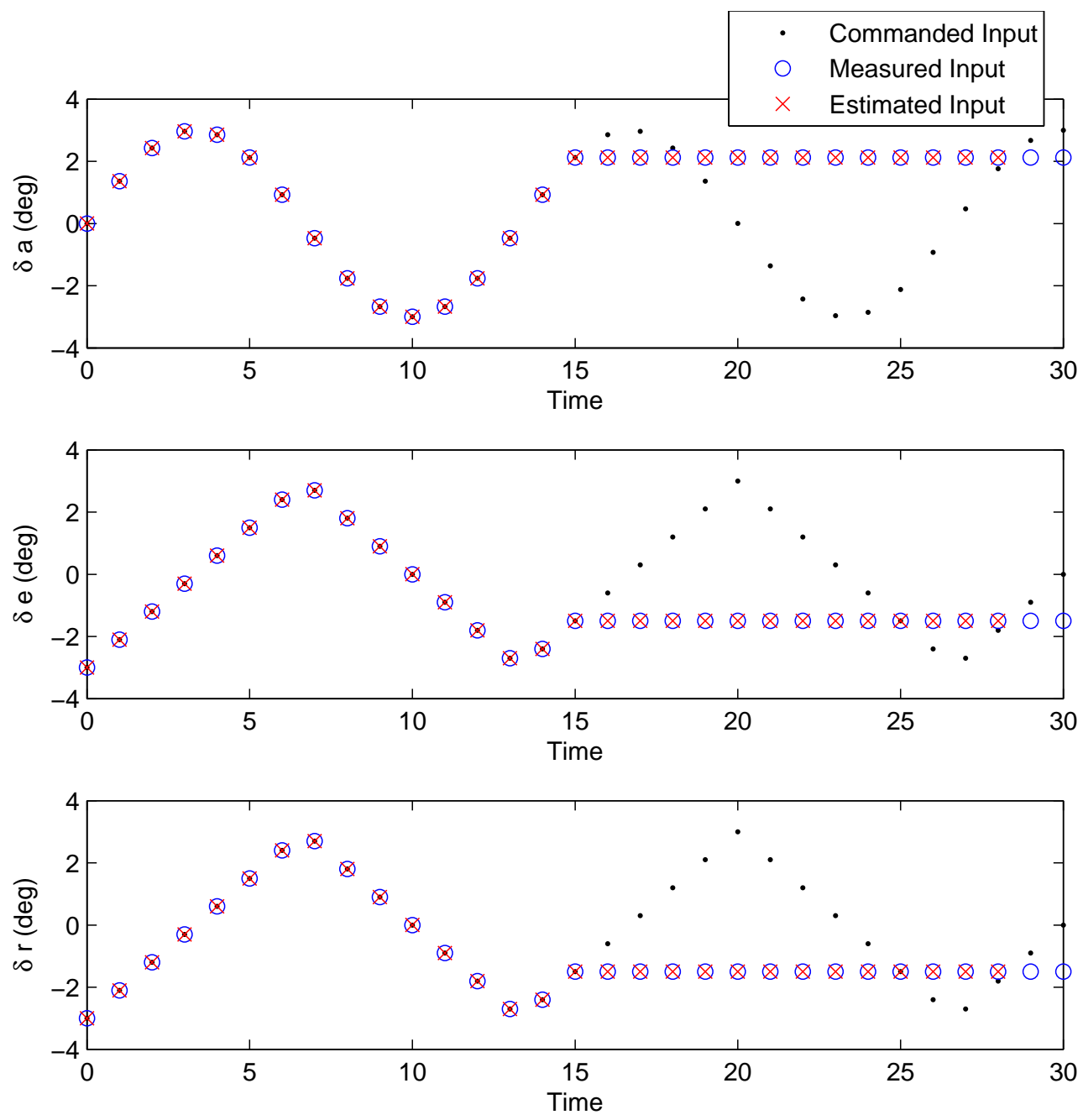

Figure 11: 2-step delay input reconstruction for GTM. Estimates of the unknown inputs $\delta a, \delta e, \delta r$ are obtained by using measurements of the outputs $u, v, p, r$ in the linearized flight model. In this example, the ailerons, elevator and, rudder become stuck at $k=16$. 

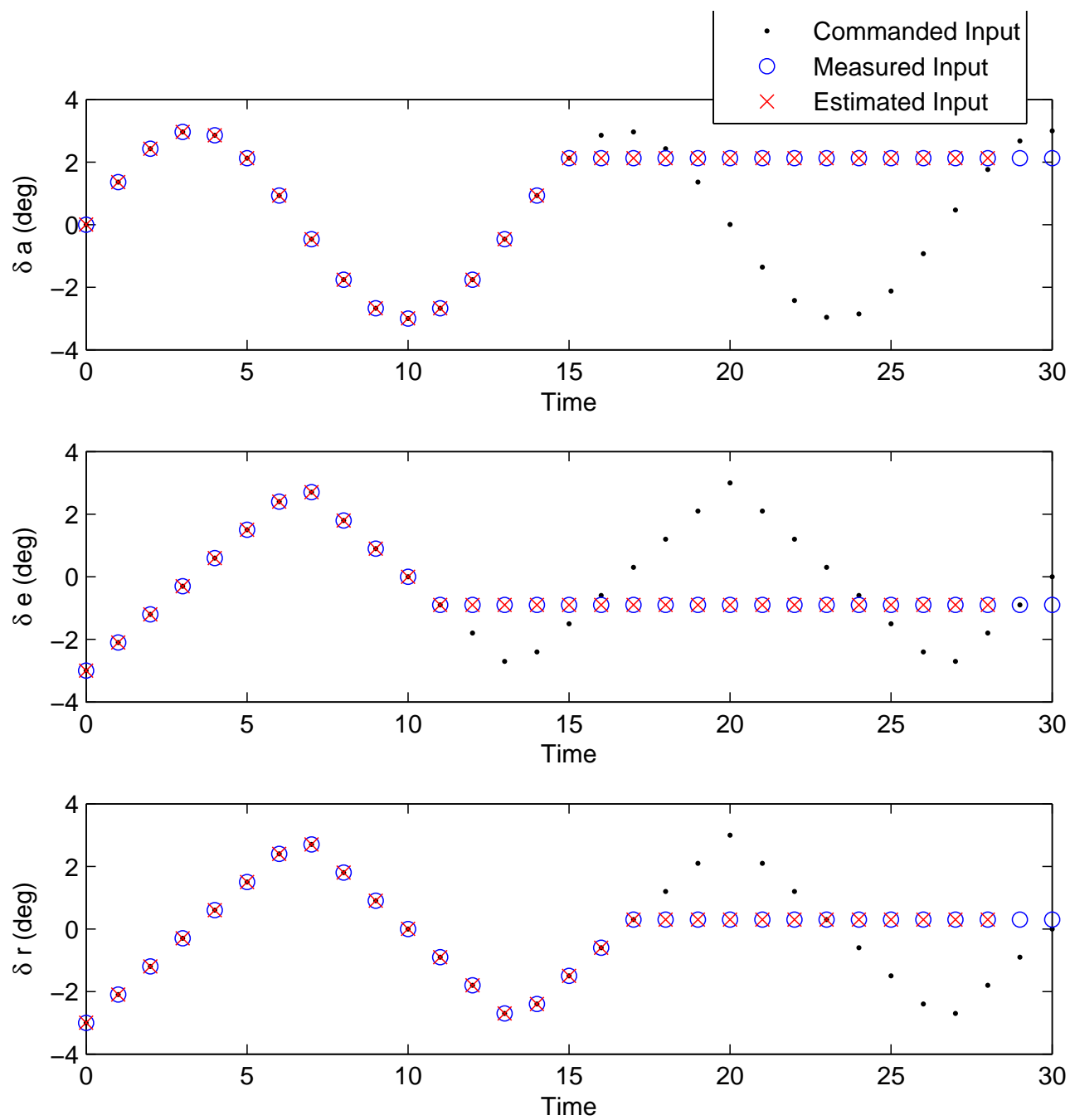

Figure 12: 2-step delay input reconstruction for GTM. Estimates of the unknown inputs $\delta a, \delta e, \delta r$ are obtained by using measurements of the outputs $u, v, p, r$ in the linearized flight model. In this example, the ailerons become stuck at $k=16$, elevator becomes stuck at $k=12$ and the rudder becomes stuck at $k=18$. 

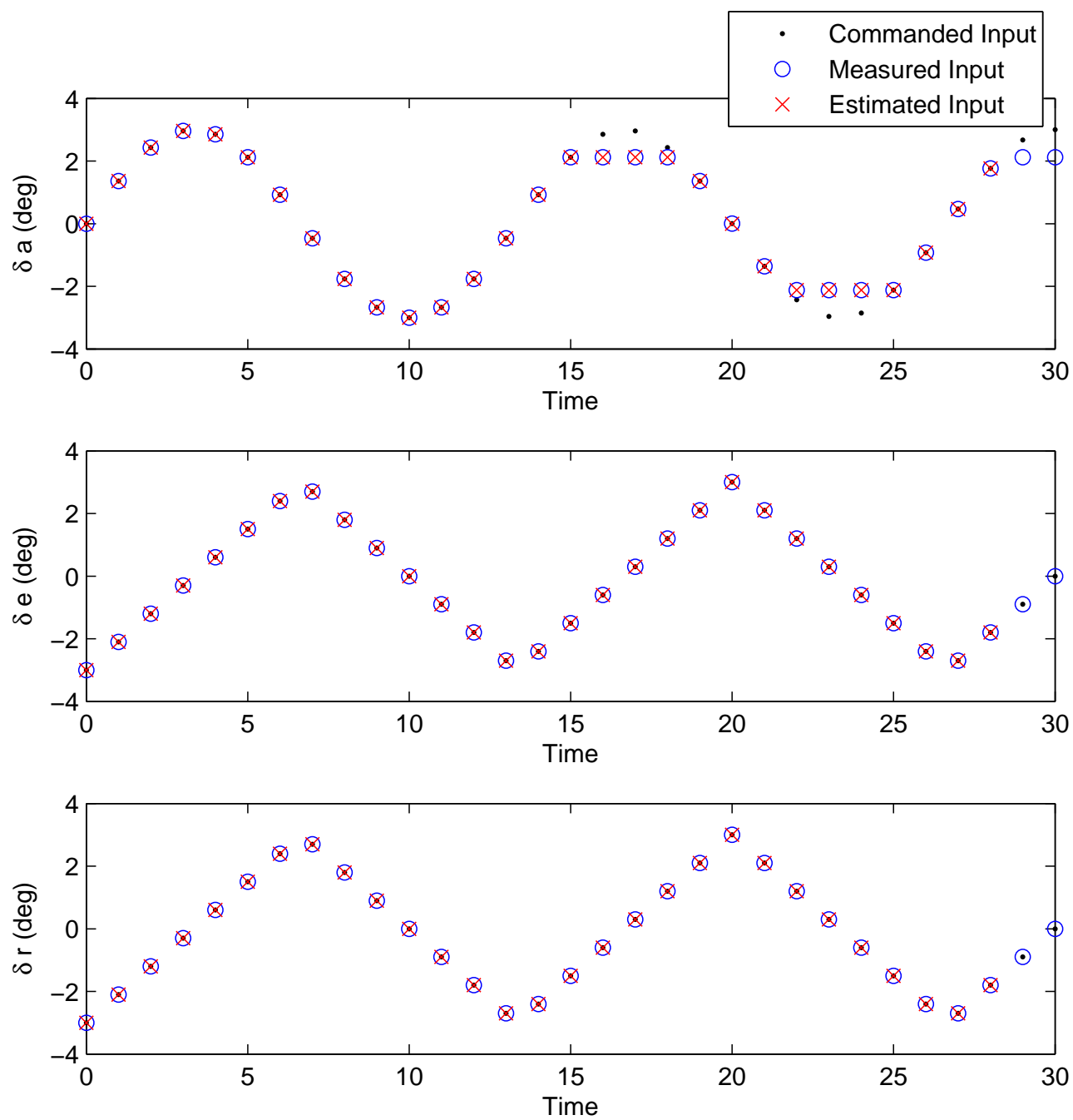

Figure 13: 2-step delay input reconstruction for GTM. Estimates of the unknown inputs $\delta a, \delta e, \delta r$ are obtained by using measurements of the outputs $u, v, p, r$ in the linearized flight model. In this example, the ailerons become subject to saturation at $k=16$. 

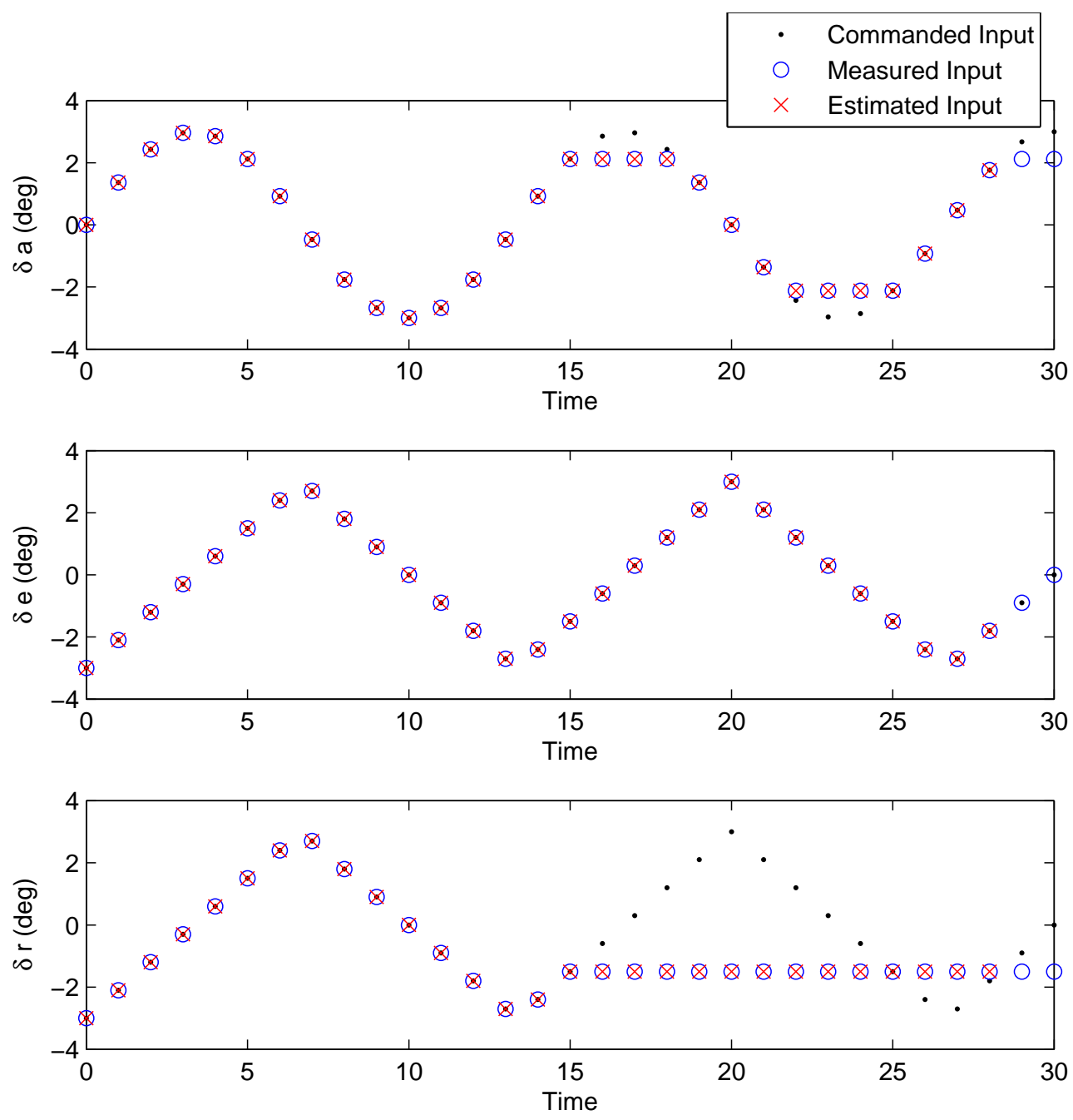

Figure 14: 2-step delay input reconstruction for GTM. Estimates of the unknown inputs $\delta a, \delta e, \delta r$ are obtained by using measurements of the outputs $u, v, p, r$ in the linearized flight model. In this example, the ailerons become subject to saturation at $k=16$ and the rudder becomes stuck at $k=16$. 

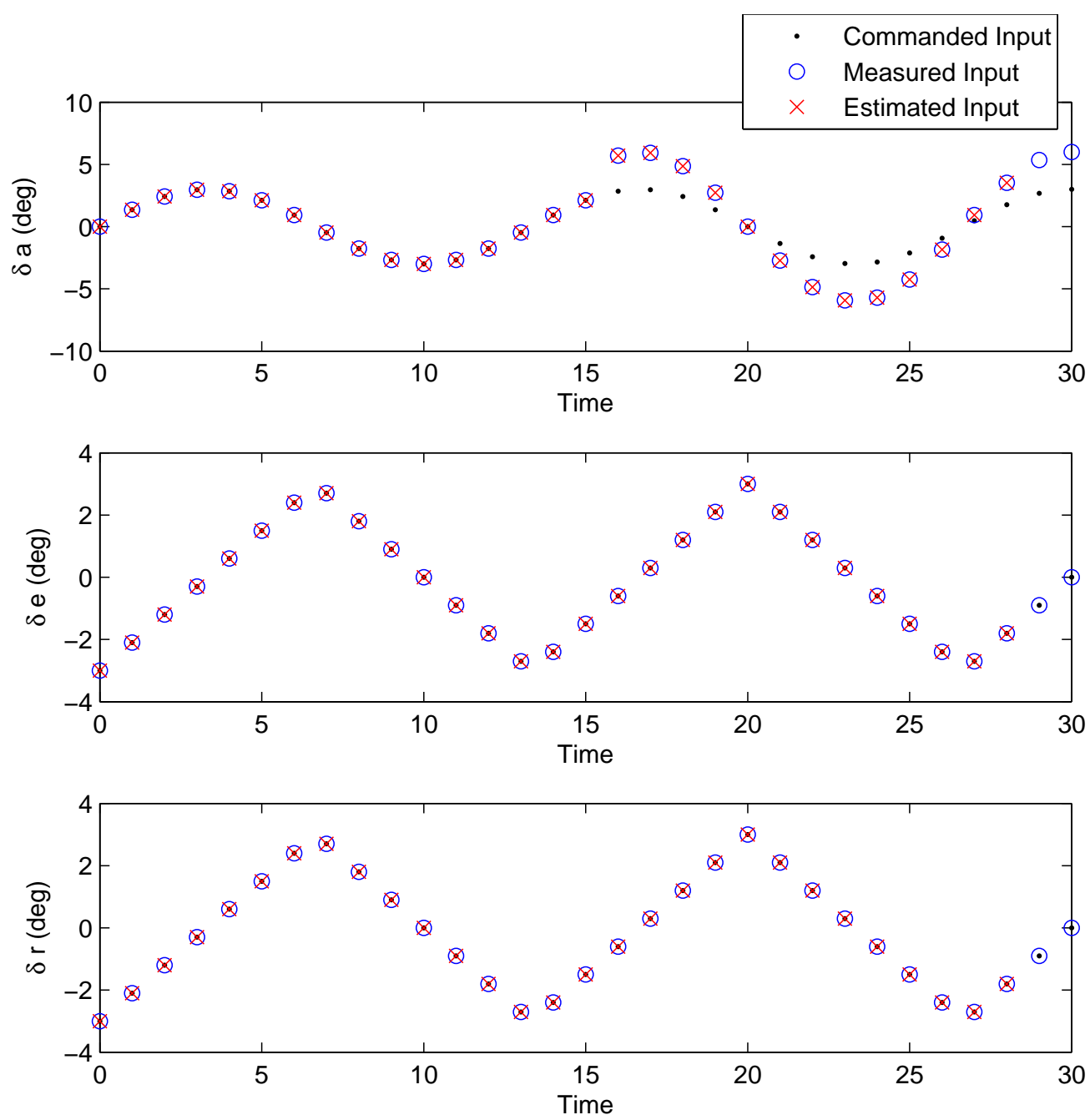

Figure 15: 2-step delay input reconstruction for GTM. Estimates of the unknown inputs $\delta a, \delta e, \delta r$ are obtained by using measurements of the outputs $u, v, p, r$ in the linearized flight model. In this example, the ailerons become subject to a scale factor error at $k=16$ by a factor of 2 . 

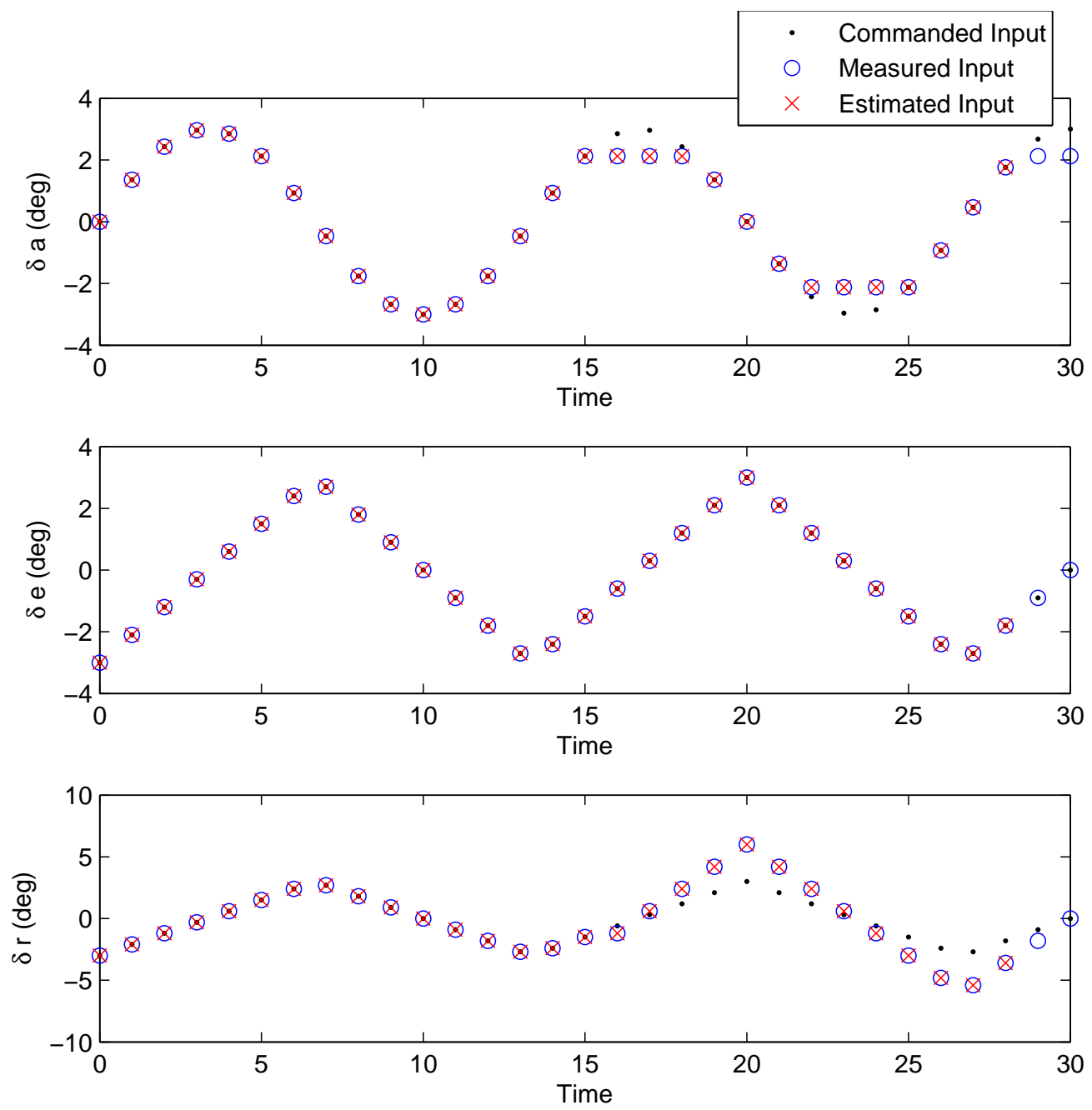

Figure 16: 2-step delay input reconstruction for GTM. Estimates of the unknown inputs $\delta a, \delta e, \delta r$ are obtained by using measurements of the outputs $u, v, p, r$ in the linearized flight model. In this example, the ailerons become subject to saturation at $k=16$, and rudder becomes subject to a scale factor error at $k=16$ by a factor of 2 . 

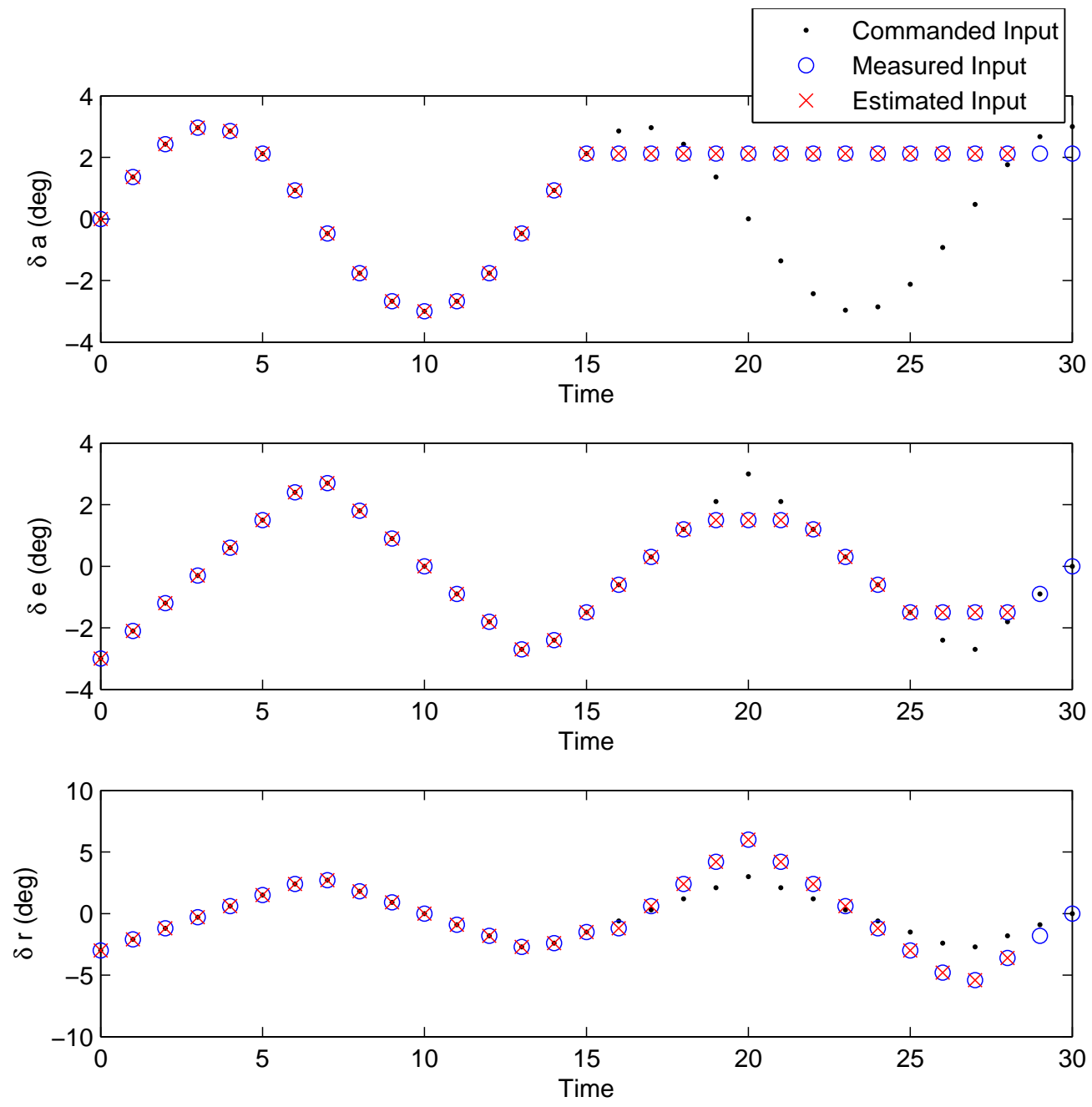

Figure 17: 2-step delay input reconstruction for GTM. Estimates of the unknown inputs $\delta a, \delta e, \delta r$ are obtained by using measurements of the outputs $u, v, p, r$ in the linearized flight model. In this example, the ailerons become stuck at $k=16$, the elevator becomes subject to saturation at $k=16$, and rudder becomes subject to a scale factor error at $k=16$ by a factor of 2 . 

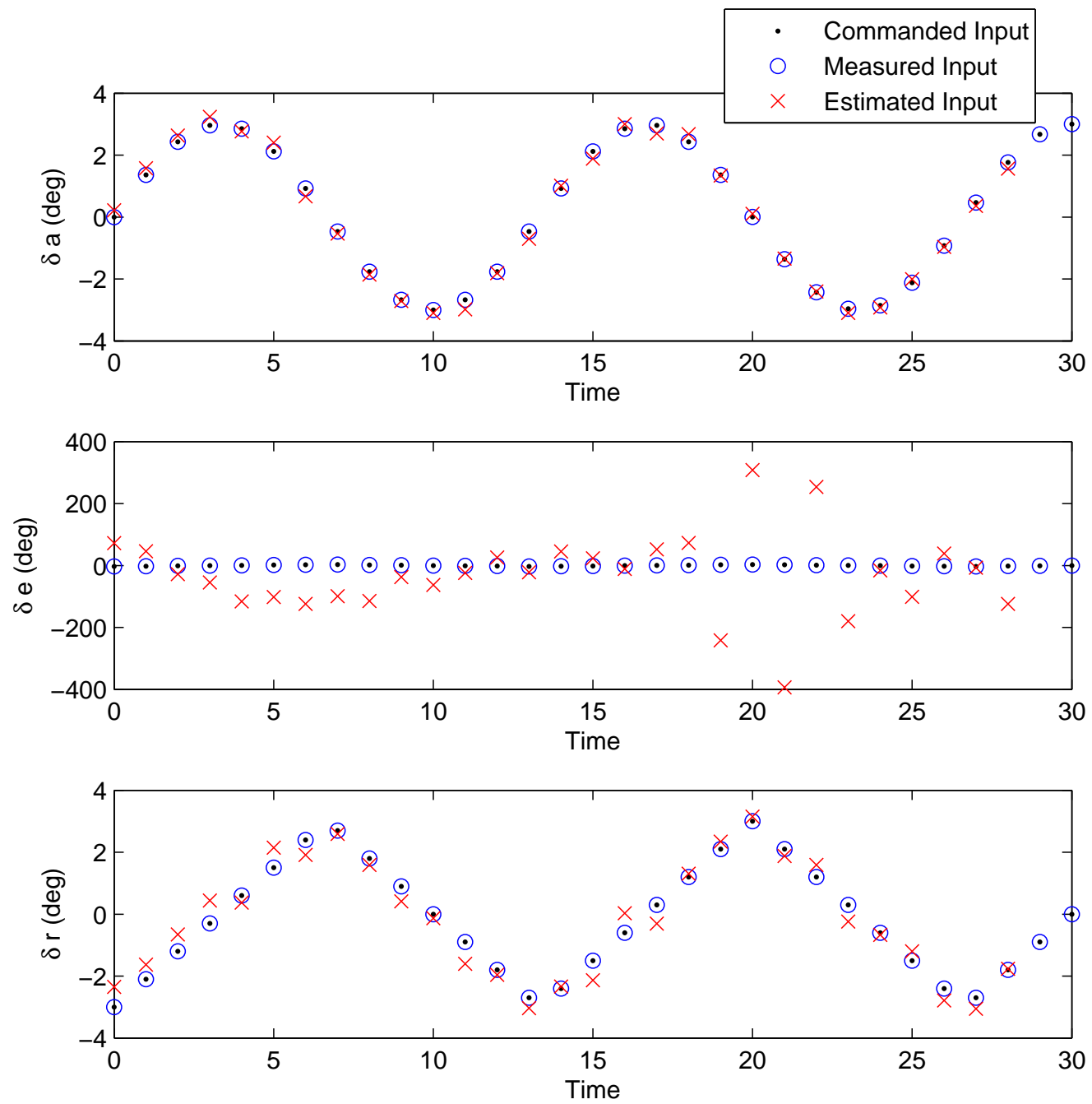

Figure 18: 2-step delay input reconstruction for GTM. Estimates of the unknown inputs $\delta a, \delta e, \delta r$ are obtained by using measurements of the outputs $u, v, p, r$ in the linearized flight model, assuming erroneous Markov parameters. 

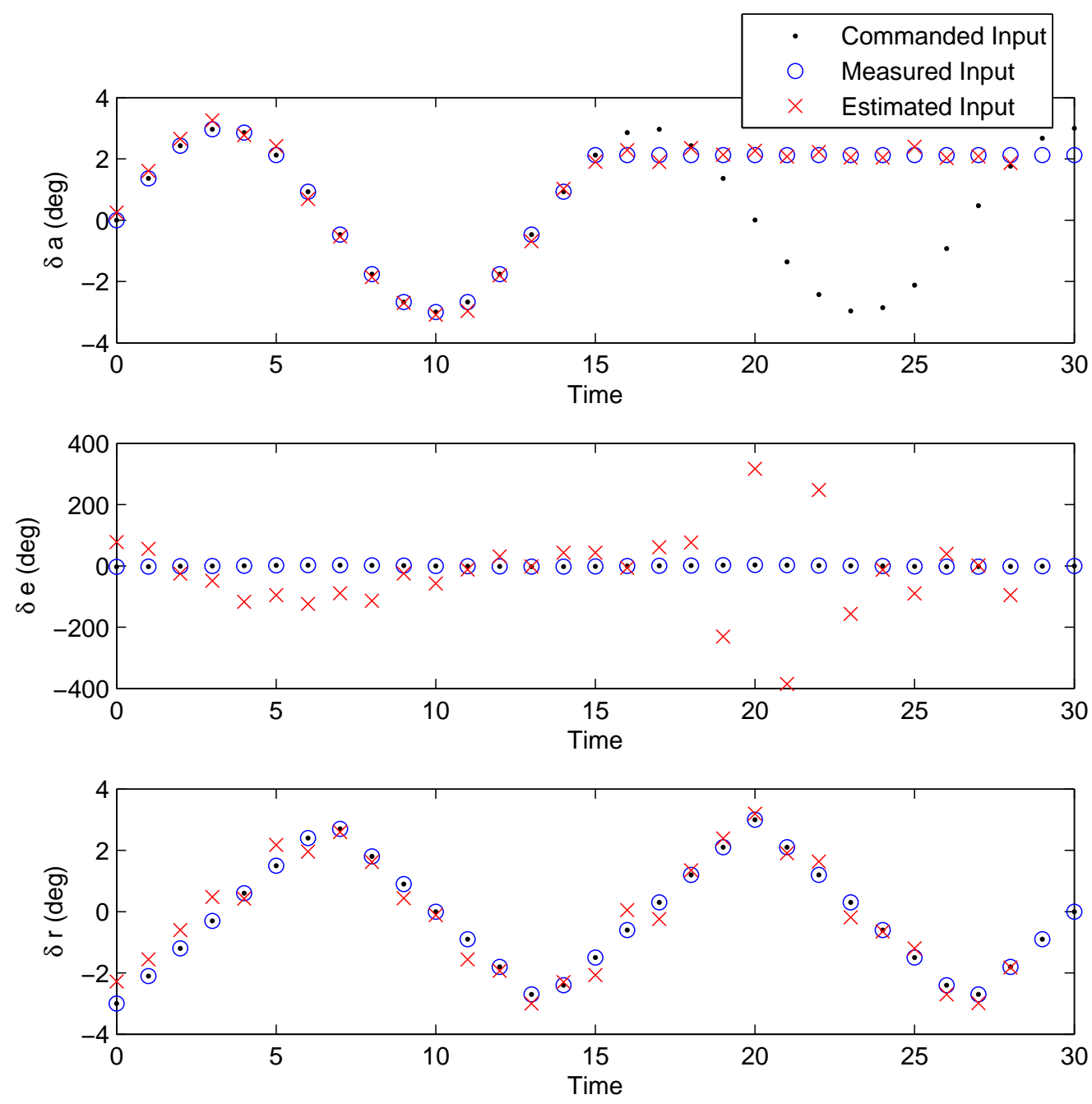

Figure 19: 2-step delay input reconstruction for GTM. Estimates of the unknown inputs $\delta a, \delta e, \delta r$ are obtained by using measurements of the outputs $u, v, p, r$ in the linearized flight model, assuming erroneous Markov parameters. In this example, the ailerons become stuck at $k=16$. 

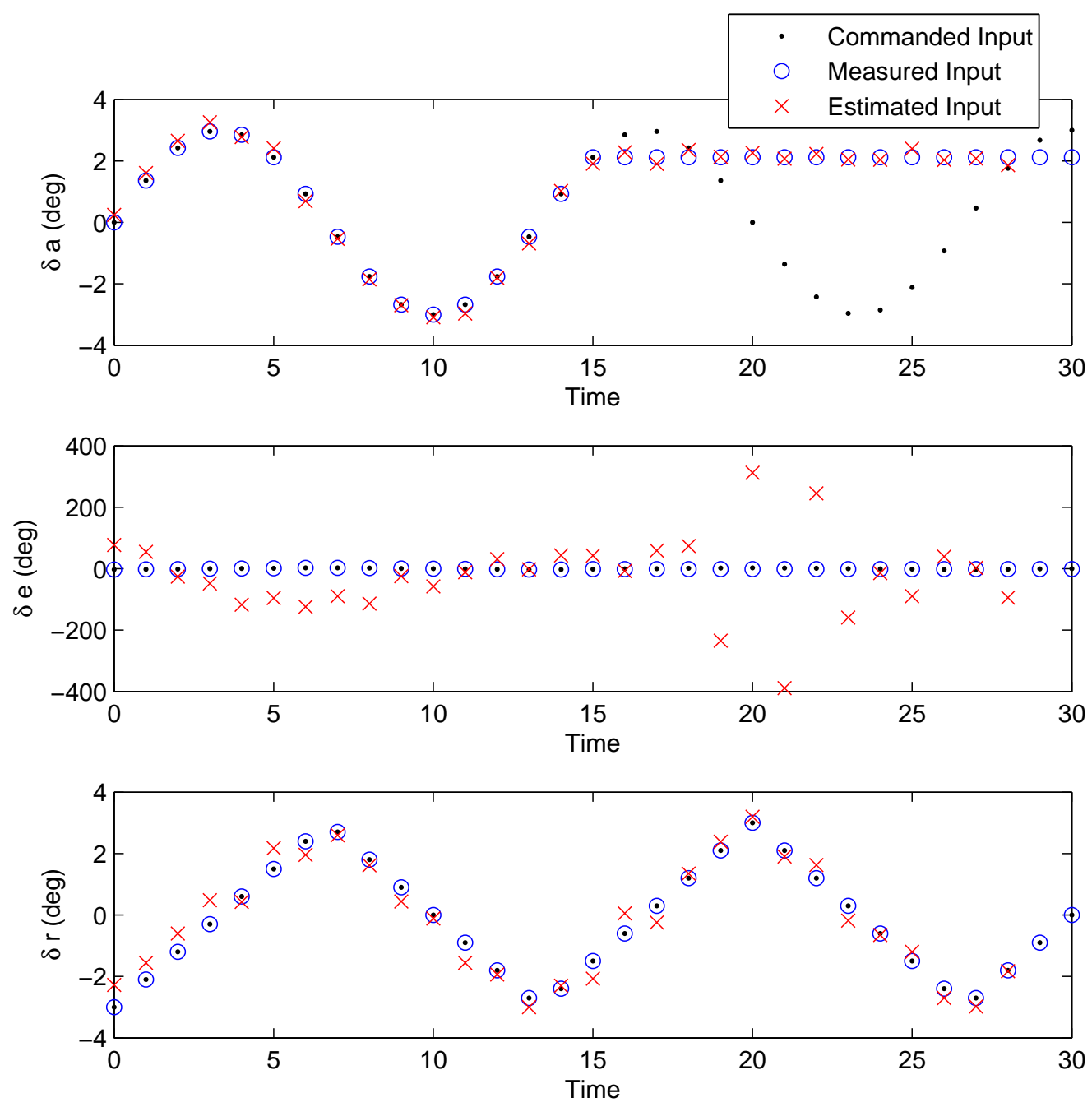

Figure 20: 2-step delay input reconstruction for GTM. Estimates of the unknown inputs $\delta a, \delta e, \delta r$ are obtained by using measurements of the outputs $u, v, p, r$ in the linearized flight model, assuming erroneous Markov parameters. In this example, the ailerons and elevator both become stuck at $k=16$. 

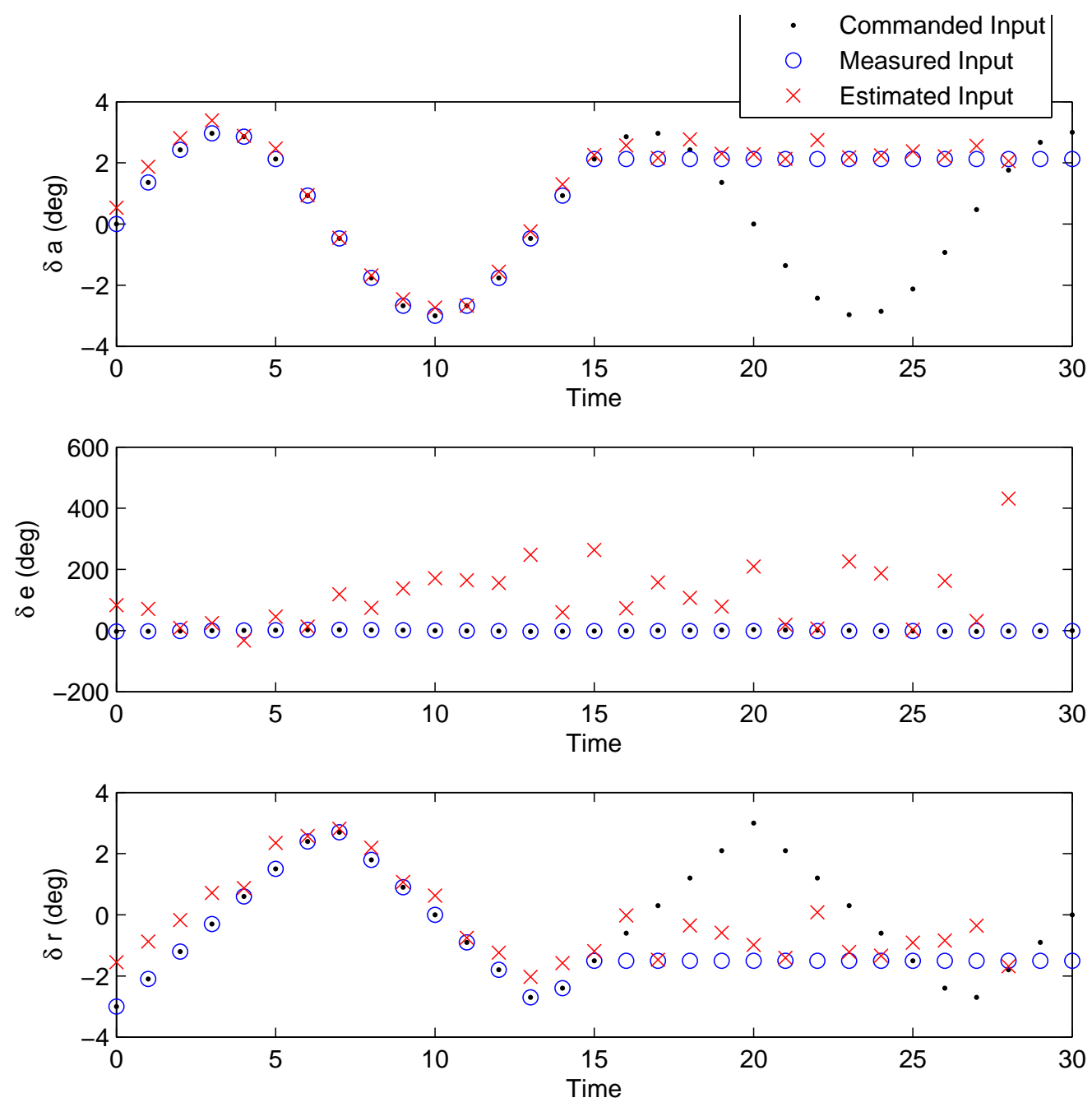

Figure 21: 2-step delay input reconstruction for GTM. Estimates of the unknown inputs $\delta a, \delta e, \delta r$ are obtained by using measurements of the outputs $u, v, p, r$ in the linearized flight model, assuming erroneous Markov parameters. In this example, the ailerons, elevator and, rudder become stuck at $k=16$. 

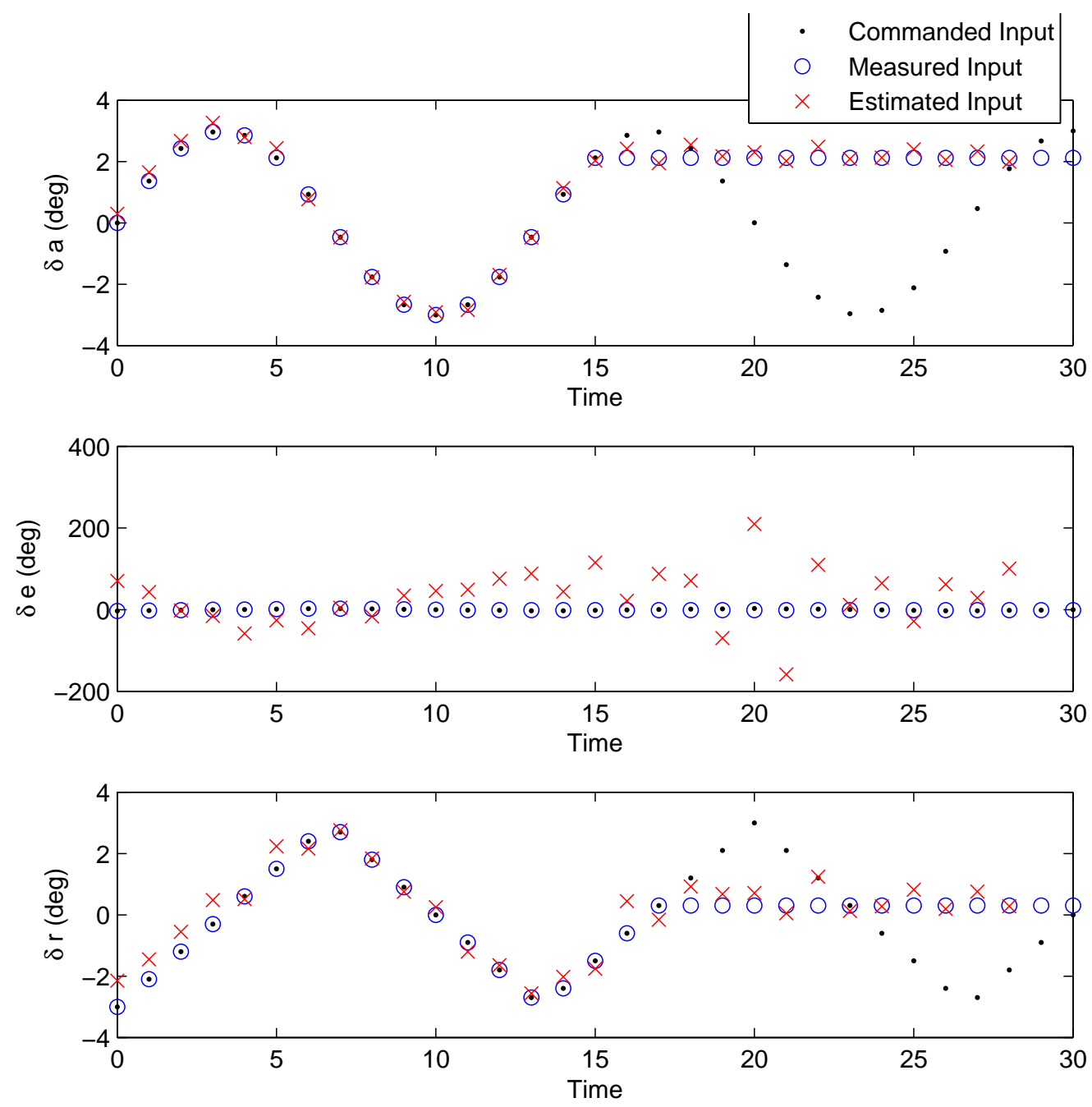

Figure 22: 2-step delay input reconstruction for GTM. Estimates of the unknown inputs $\delta a, \delta e, \delta r$ are obtained by using measurements of the outputs $u, v, p, r$ in the linearized flight model, assuming erroneous Markov parameters. In this example, the ailerons become stuck at $k=16$, elevator becomes stuck at $k=12$ and the rudder becomes stuck at $k=18$. 

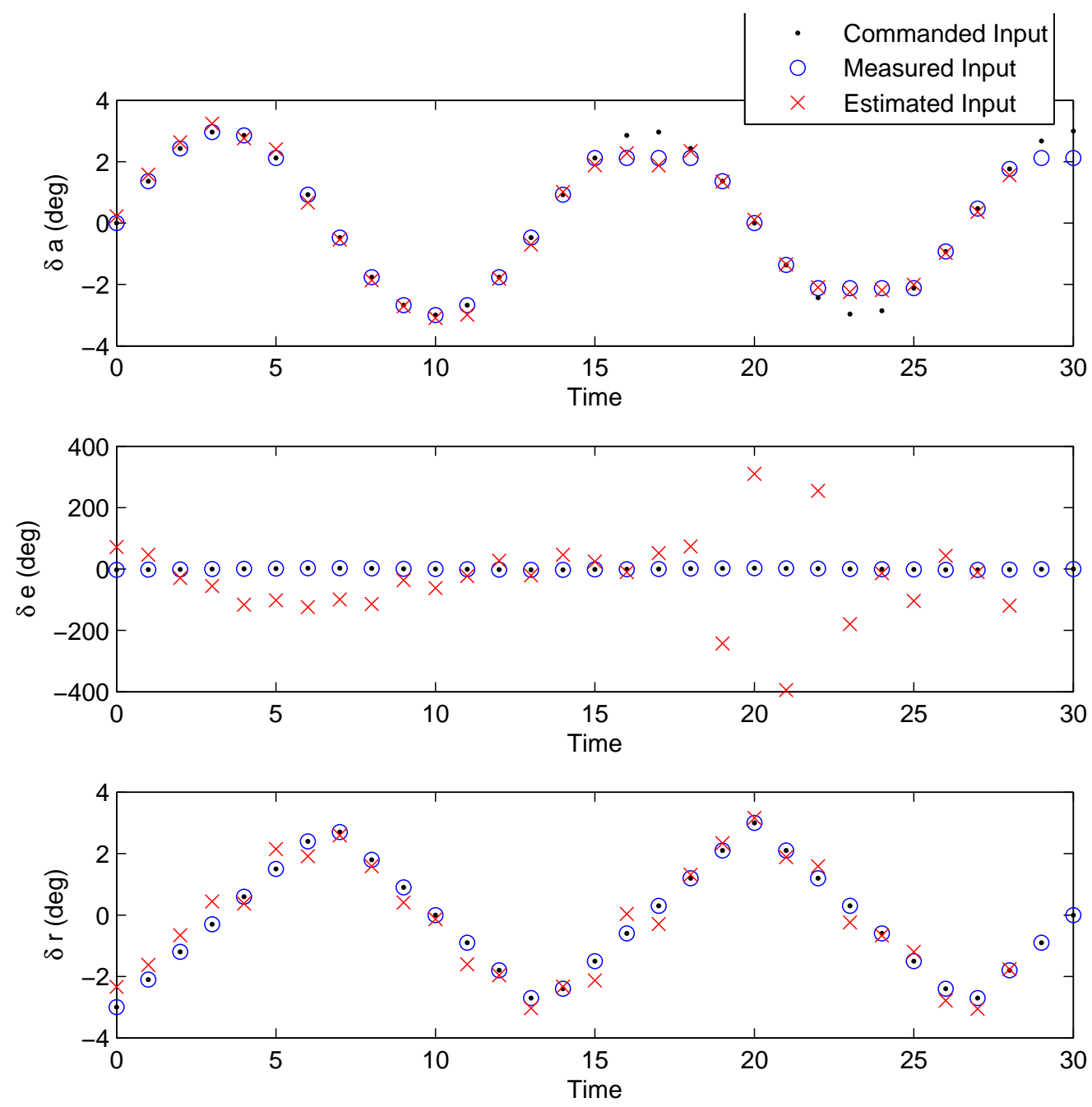

Figure 23: 2-step delay input reconstruction for GTM. Estimates of the unknown inputs $\delta a, \delta e, \delta r$ are obtained by using measurements of the outputs $u, v, p, r$ in the linearized flight model, assuming erroneous Markov parameters. In this example, the ailerons become subject to saturation at $k=16$. 

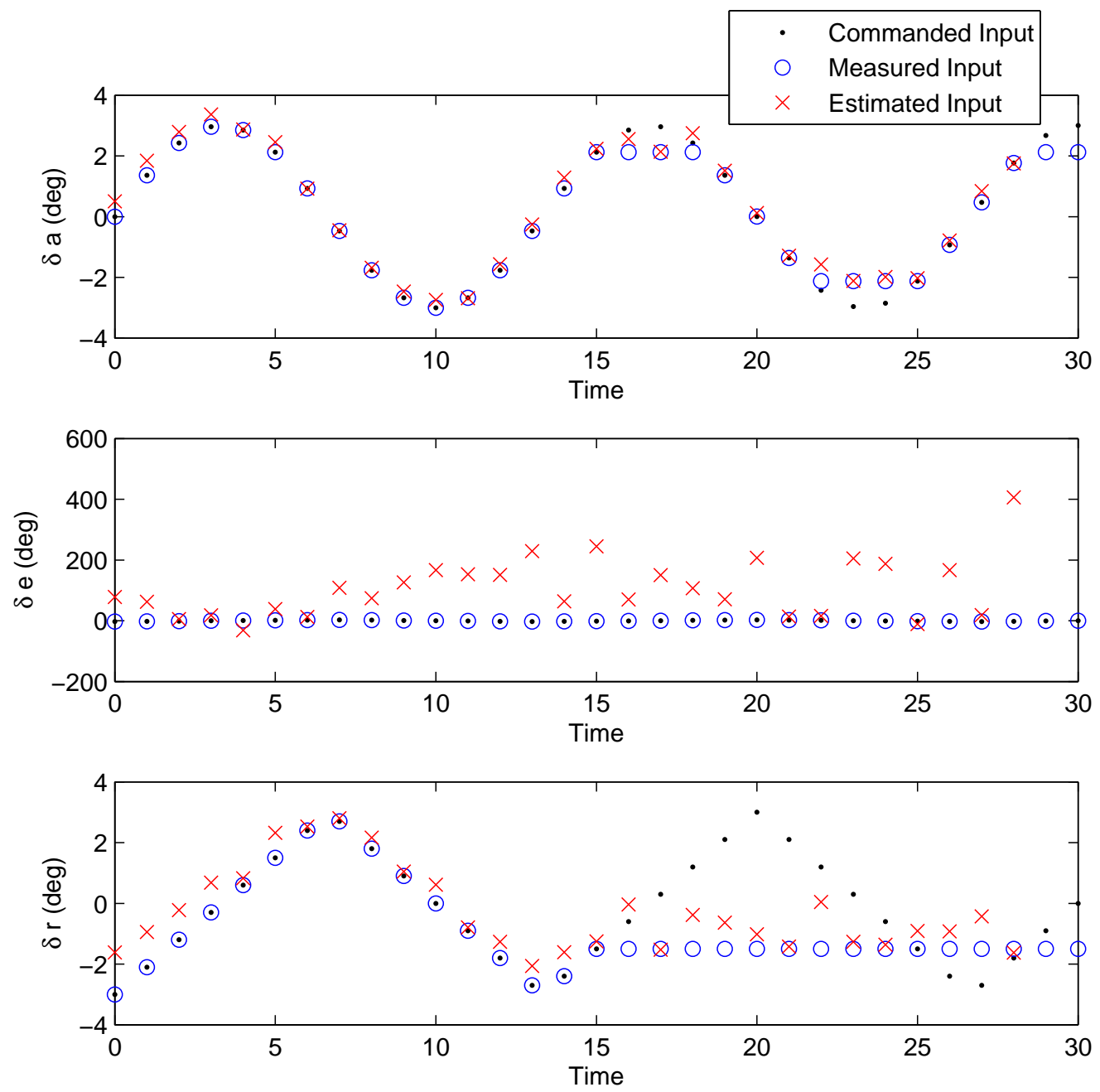

Figure 24: 2-step delay input reconstruction for GTM. Estimates of the unknown inputs $\delta a, \delta e, \delta r$ are obtained by using measurements of the outputs $u, v, p, r$ in the linearized flight model, assuming erroneous Markov parameters. In this example, the ailerons become subject to saturation at $k=16$ and the rudder becomes stuck at $k=16$. 

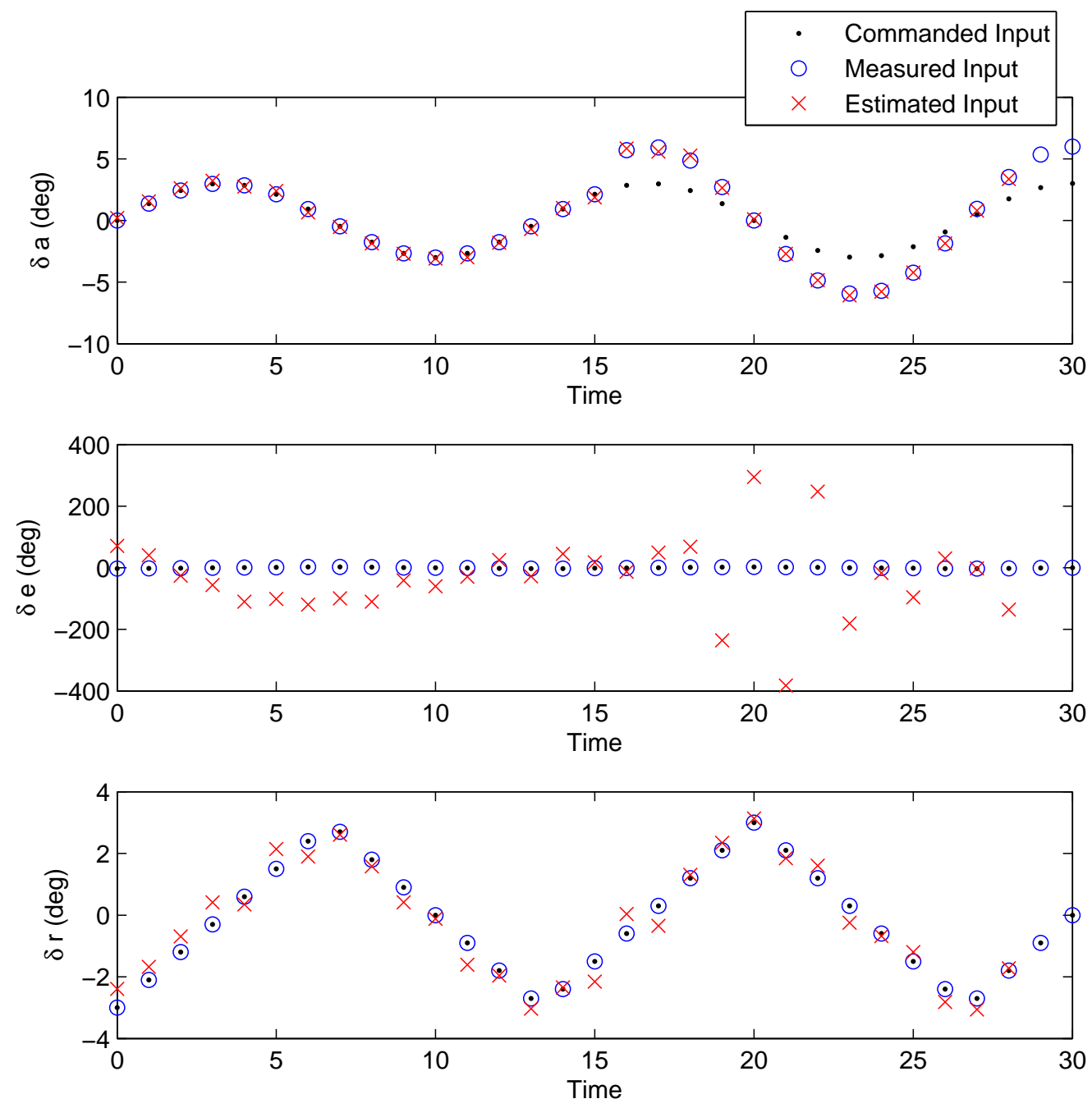

Figure 25: 2-step delay input reconstruction for GTM. Estimates of the unknown inputs $\delta a, \delta e, \delta r$ are obtained by using measurements of the outputs $u, v, p, r$ in the linearized flight model, assuming erroneous Markov parameters. In this example, the ailerons become subject to a scale factor error at $k=16$ by a factor of 2 . 
${ }^{7}$ M. K. Sain and J. L. Massey. Invertibility of linear time-invariant dynamical systems. IEEE transactions on automatic control, AC-14:141-149, 1969.

${ }^{8} \mathrm{M}$. Tomizuka. Zero phase error tracking algorithm for digital control. ASME Journal of Dynamic Systems, Measurement, and Control, 109:65-68, 1987.

${ }^{9}$ E. Gross and M. Tomizuka. Experimental flexible beam tip tracking control with a truncated series approximation to uncancelable inverse dynamics. IEEE transactions on control systems technology, 2:382-391, 1994.

${ }^{10} \mathrm{E}$. Gross, M. Tomizuka, and W. Messner. Cancellation of discrete time unstable zeros by feedforward control. Journal of Dynamic Systems, Measurement, and Control, 116:33-38, 1994.

${ }^{11}$ J. Chen, R. J. Patton, and H.-Y. Zhang. Design of unknown input observers and robust fault detection filters. Int. J. Contr., 63:85-105, 1996.

${ }^{12}$ M. Hou and R.J. Patton. Input observability and input reconstruction. Automatica, 34:789-794, 1998.

${ }^{13}$ G. Marro, D. Prattichizzo, and E. Zattoni, "A unified algorithmic setting for signal-decoupljing compensators and unknown-input observers," Proc. Conf. Dec. Contr., pp. 4512-4517, Sydney, Australia, 2000.

${ }^{14}$ G. Marro, D. Prattichizzo, and E. Zattoni, "Convolution proiles for right inversion of multivariable non-minimum phase discrete-time systems," Automatica, 38: 1695-1703, 2002.

${ }^{15}$ S. Gillijns. Kalman Filtering Techniques for System Inversion and Data Assimilation. PhD thesis, 2007.

${ }^{16}$ J. A. Butterworth, L. Y. Pao, and D. Y. Abramovitch. The effect of nonminimum-phase zero locations on the performance of feedforward model-inverse control techniques in discrete-time systems. 2008 American Control Conference, 2008.

${ }^{17}$ H. J. Palanthandalam-Madapusi and D. S. Bernstein, A Subspace Identification Algorithm for Simultaneous Identification and Input Reconstruction. Proc. Conf. Dec. Contr., pp. 4956-4961, New Orleans, LA, December, 2007; Int. J. Adapt. Control Signal Process, to appear.

${ }^{18}$ H.-Y. Fu, J. Yan, M. A. Santillo, H. Palanthandalam-Madapusi, and D. S. Bernstein Fault Detection for Aircraft Control Surfaces Using Approximate Input Reconstruction. Proc. Amer. Contr. Conf.,St. Louis, MO, June 2009.

${ }^{19}$ J.-N. Juang, Applied System Identification, Prentice-Hall, 1993.

${ }^{20}$ Bailey, R., Hostetler, R., Barnes, K., Belcastro, C., and Belcastro, C., "'Experimental Validation: Subscale Aircraft Ground Facilities and Integrated Test Capability," Proc. Guid. Nav. Contr. Conf., San Francisco, CA, August 2005, AIAA2005-6433.

${ }^{21}$ Jordan, T. and Bailey, R., "'NASA Langley's AirSTAR Testbed: A Subscale Flight Test Capability for Flight Dynamics and Control System Experiments," Proc. Guid. Nav. Contr. Conf., Honolulu, HI, August 2008, AIAA-2008-6660.

${ }^{22}$ Murch, A., "A Flight Control System Architecture for the NASA AirSTAR Flight Test Infrastructure," Proc. Guid. Nav. Contr. Conf., Honolulu, HI, August 2008, AIAA-2008-6990. 